(E,Z)-Gleichgewichte, $15^{[1]}$

\title{
Synthesen und erhöhte Konfigurationslabilität von 2-Iminoindan-Derivaten mit Vorderseitenspannung
}

\author{
Rudolf Knorr*a , Kathrin Ferchland ${ }^{\text {a }}$, Johann Mehlstäubl ${ }^{a}$, Thi Phung Hoang ${ }^{\text {a }}$, Petra Böhrer ${ }^{\text {, }}$, Hans-Dietrich Lüdemann ${ }^{b}$ \\ und Elmar Lang ${ }^{b}$
}

Institut für Organische Chemie der Universität München ${ }^{a}$, Karlstraße 23, W-8000 München 2

Institut für Biophysik und physikalische Biochemie der Universität Regensburg ${ }^{\mathrm{b}}$, Universitätsstraße 31, W-8400 Regensburg

Received January 15, 1992

Key Words: Basicity / Diastereotopomerization, $(E / Z)$ / Imines / Steric shielding / Steric strain

\section{$(E, Z)$ Equilibria, $15^{[1]}$ - Syntheses and Lability of the Configuration of 2-Iminoindan Derivatives with Front Strain}

Syntheses and properties are described for sterically shielded imines $\mathrm{R}_{2} \mathrm{C}=\mathrm{NR}(\mathbf{3 c}, \mathbf{e}, \mathbf{g}-\mathbf{k})$, which are rather inert toward nucleophiles. Nucleophilic attack at the nitrogen atom of $\mathbf{3 k}$ is indicated by the formation of the azine 4 . $(E, Z)$ Configurational diastereotopomerization (anti/syn) is strongly dependent on $\mathrm{N}$ substituents $\left[\mathrm{CH}_{3}\right.$, phenyl, 1-naphthyl, acetyl, $\mathrm{Si}\left(\mathrm{CH}_{3}\right)_{3}$, cyano, $\mathrm{SC}_{6} \mathrm{H}_{5}, \mathrm{SOC}_{6} \mathrm{H}_{5}, \mathrm{SO}_{2} \mathrm{C}_{6} \mathrm{H}_{5}$, and nitro]. It is accelerated by front strain along the $\mathrm{C}=\mathrm{N}$ bond in $\mathbf{3 a}-\mathbf{c}, \mathbf{e}, \mathbf{g}, \mathbf{h}, \mathbf{j}$, and $\mathbf{k}$ and provides an energetic basis (by $\Delta G^{+}$and $\Delta \Delta G^{+}$values) for the gauging of force-field parameters. This $(E, Z)$ stereomutation is characterized by a vanishing solvent dependence and a positive volume of activation $\left(+10.2 \mathrm{~cm}^{3} \mathrm{~mol}^{-1}\right.$ for $\left.3 a\right)$. In contrast to other $\pi$ acceptor substituents, the $N$-nitro group in 31 retards $(E, Z)$ interconversion.
Imino-Derivate von Aldehyden oder von „unsymmetrischen“ Ketonen können in zwei diastereomeren Formen $(E)-1$ und $(Z)-1$ $(X \neq Y)$ auftreten. Diese geometrische Stereoisomerie verschwindet mit konstitutionsgleichen Substituenten $\mathrm{X}=\mathrm{Y}$, die in 1 diastereotop sind (syn und anti) und sich deswegen NMR-spektroskopisch unterscheiden lassen. Die Mobilität der $(E, Z)$-Konfigurationsumwandlung (Diastereotopomerisierung, wenn $X=Y$ ) hängt stark $^{[2-4]}$ von den Substituenten $R$ ab. Erneute Hinweise auf gelegentlich ähnliche Substituentenabhängigkeiten fanden wir bei gewissen $^{[5-7]}$ Vinyllithiumderivaten 2, deren Carbanion-Teile als die isoelektronischen Analoga ${ }^{4,8]}$ von Iminen 1 aufgefaßt werden können. Sperrige Reste $\mathrm{X}$ und $\mathrm{Y}$ in 2 beschleunigen die Diastereotopomerisierung; am mechanistisch einfacheren Modell der Imine 1 ließe sich die sterisch verursachte Beschleunigung aber klarer als energetische Destabilisierung des Grundzustands interpretieren. $\mathrm{Um}$ in dieser Weise die intramolekular abstoßenden Wechselwirkungen zu quantifizieren, haben wir die Imine $3 \mathbf{a}-1$ hergestellt und NMR-spektroskopisch nach den Geschwindigkeitskonstanten ihrer $(E, Z)$-Topomerisierung gesucht. Bei der mit $\mathbf{3 b}$ verwandten SchiffBase 3,5-Dimethyl-4-[(2,2,5,5-tetramethylcyclopentyliden)amino]phenol hatte die Röntgenstrukturanalyse ${ }^{[9]}$ bereits geometrische Hinweise auf die Vorderseitenspannung längs der CN-Doppelbindung ergeben.

\section{A. Synthesen und Hydrolysestabilität der Imine}

Zur Herstellung des $N$-(1-Naphthyl)imins $3 c$ aus 1,1,3,3Tetramethyl-2-indanon verwendet man am besten das Ti(IV)-Salz des 1-Aminonaphthalins. Dagegen reagiert das sterisch behinderte 1-Amino-2-methylnaphthalin selbst unter derartig verschärften Bedingungen weder mit jenem Keton noch mit dem $N$-unsubstituierten Imin 3d. Dieses Imin<smiles>[X]C([Y])=[N+]([R])[O-]</smiles><smiles>[X]C([Y])=[N+]([R])[O-]</smiles>

$1(E)$
$1(Z)$<smiles>[X]C([Y])=C([R])[AlH2]</smiles>

$2(Z)$

ist durch Reduktion ${ }^{[1]}$ des entsprechenden Oxims oder durch Ammonolyse $^{[10]}$ des Nitro-imins 3l gut zugänglich. Die $N$-Acetylverbindung 3 e erhält man aus $3 \mathbf{d}$ mit Acetylchlorid und Pyridin in Diethylether und daneben in äquivalenter Ausbeute 3d-Hydrochlorid ${ }^{[1]} ; \mathbf{3 d}$ ist also in diesem Lösungsmittel wie in Chloroform ${ }^{[1]}$ basischer als Pyridin. Nach der Schotten-Baumann-Reaktion mit Acetylchlorid in wäßrigalkalischer Suspension wird 3d unverändert zurückgewonnen. Im Gegensatz zu einigen der bekannten ${ }^{[1-13]} N$-Acylimine ist $3 \mathbf{e}$ gegen verd. Natronlauge tagelang stabil. Aus $\mathrm{CCl}_{4}$-Lösung wird $3 \mathrm{e}$ durch $2 \mathrm{~N} \mathrm{HCl}$ nicht extrahiert, doch hydrolysieren in diesem Zweiphasensystem während eines Tages etwa $20 \%$ 3e zu 1,1,3,3-Tetramethyl-2-indanon.

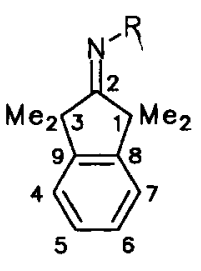

3

\begin{tabular}{c|c|c|c} 
3 & R & 3 & R \\
\hline a & Methyl & g & SiMe3 \\
b & Phenyl & h & Cyan \\
c & 1-Naphthyl & i & S-Ph \\
d & H & j & SO-Ph \\
e & Acetyl & k & SO $_{2}-\mathrm{Ph}$ \\
f & Li & l & Nitro
\end{tabular}


Das bereits beschriebene ${ }^{[1]} N$-Lithio-Derivat $3 \mathrm{f}$ wird am besten in THF-Lösung zu $\mathbf{3 g}$, h oder $\mathbf{i}$ umgesetzt, wobei $\mathbf{3 f}$ aus 3d mit $n$-Butyllithium hergestellt werden kann. Zur Synthese des labilen $N$-Trimethylsilyl-Derivats $\mathbf{3 g}$ ist aber die einleitende Titration ${ }^{[1]}$ von 3d mit Methyllithium bis zum Ende der Methan-Entwicklung (Gasbürette) vorzuziehen; denn nur bei vollständiger Bildung von $\mathbf{3 f}$ läßt sich die Verunreinigung des Produkts $3 g$ durch das schwer abtrennbare Edukt 3d in Grenzen halten. Da $3 \mathrm{~g}$ durch neutrales Wasser augenblicklich zum Imin 3d hydrolysiert wird, darf das Reaktionsgemsich nur durch Eingießen in überschüssige verd. Lauge aufgearbeitet und nicht mit NatriumcarbonatLösung oder Wasser gewaschen werden; es enthält dann neben 3d erstaunlicherweise auch das bekannte ${ }^{[14,15]}$ Azin 4. Bei der Behandlung mit Chlortrimethylsilan und Triethylamin bleibt das Imin 3d unverändert.

Schon ohne Optimierung der Bedingungen erhält man aus $3 \mathrm{f}$ akzeptable Ausbeuten an $\mathbf{3 h}$ mit Tosylcyanid oder an 3i mit Diphenyldisulfid. Beide Produkte sind stundenlang gegen alkalisches und saures Wasser bei Raumtemperatur stabil; als nicht-basische Substanzen lassen sie sich leicht vom unverbrauchten Imin 3d abtrennen. Auch bei der Umsetzung von 3d mit Benzolsulfenylchlorid ${ }^{[16]}$ erhält man das $N$-(Phenylthio)imin 3i. Es reagiert nicht mit 30proz. Wasserstoffperoxid in Chloroform-Emulsion bei $+50^{\circ} \mathrm{C}$, bildet aber mit $m$-Chlorperbenzoesäure viel $N$-Sulfonyl-Derivat 3k neben dem Edukt 3i und wenig $N$-Sulfinyl-Produkt 3j. Die selektive Oxidation von 3i zu 3j gelingt mit dem Mg-Salz der Monoperoxyphthalsäure ${ }^{[17]}$ unter Phasentransferkatalyse und Eiskühlung in wenigen Minuten; beim Erwärmen kann auch hier Weiteroxidation eintreten. Mit $\mathrm{m}$ Chlorperbenzoesäure liefert $\mathbf{3 j}$ ausschließlich $3 \mathbf{k}$ (also keine $\mathrm{N}$-Oxidation), das auch aus dem Imin 3d mit Benzolsulfonylchlorid erhalten wird. Nach der Umsetzung des $\mathrm{N}$-Lithio-Derivats $3 \mathrm{f}$ mit Benzolsulfonylchlorid oder nach inversem Zusammengeben dieser Reaktanten läßt sich dagegen nur das nicht basische Azin $^{[14,15]} 4$ isolieren. Zunächst entsteht dabei vermutlich $\mathbf{3 k}$ und reagiert dann schnell mit $\mathbf{3 f}$, wie die Isolierung von $\mathbf{4}$ aus dem Kontrollexperiment mit 3f und 3k zeigte. Aus $\mathrm{CDCl}_{3}$-Lösung lassen sich $\mathbf{3 j}$ und $\mathbf{k}$ nicht mit verd. Säure extrahieren; sie werden dabei aber während einiger Stunden zu 1,1,3,3-Tetramethyl-2-indanon hydrolysiert.

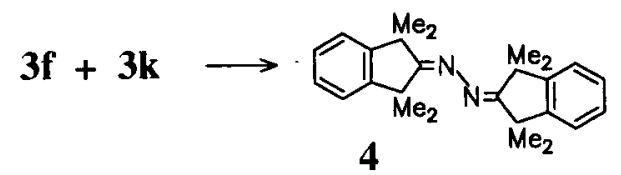

Die Zuordnung der ${ }^{13} \mathrm{C}$-NMR-Absorptionen in Tab. 1 folgt aus den Signalmultiplizitäten bei der Aufnahme ${ }^{1} \mathrm{H}$ -

Tab. $1 .{ }^{13} \mathrm{C}$ - und einige ${ }^{1} \mathrm{H}-\mathrm{NMR}$-Verschiebungen $(\delta)$ der Imine 3 und 4 in $\mathrm{CDCl}_{3}$

\begin{tabular}{|c|c|c|c|c|c|c|c|c|c|}
\hline $\mathbf{R}=$ & $\begin{array}{c}\text { 3c } \\
\text { 1-Naph }\end{array}$ & $\begin{array}{c}3 e \\
\mathrm{COCH}_{3}\end{array}$ & $\begin{array}{c}\mathbf{3 g} \\
\mathrm{SiMe}_{3}\end{array}$ & $\begin{array}{l}\text { 3h } \\
\mathrm{CN}\end{array}$ & $\begin{array}{c}\mathbf{3 i} \\
\mathrm{SPh}\end{array}$ & $\begin{array}{l}\mathbf{3 j} \\
\text { SOPh }\end{array}$ & $\begin{array}{c}3 \mathbf{k} \\
\mathrm{SO}_{2} \mathrm{Ph}\end{array}$ & $\begin{array}{c}31 \\
\mathrm{NO}_{2}\end{array}$ & $\begin{array}{c}4 \\
\text { Azin }\end{array}$ \\
\hline Temp. $\left[{ }^{\circ} \mathrm{C}\right]$ & -60 & -85 & $-71[a]$ & -34 & +23 & +9 & -71 & +29 & +25 \\
\hline $1-\mathrm{CH}_{3}$ & $29.1,30.3$ & 26.8 & 28.3 & 25.6 & 24.2 & 29.2 & 26.8 & 27.9 & 26.6 \\
\hline$H$ & $1.13^{[b]}$ & 1.49 & 1.23 & 1.69 & 1.61 & {$[c]$} & $1.83^{[\mathrm{e}]}$ & 1.55 & 1.63 \\
\hline $3-\mathrm{CH}_{3}$ & $25.7,31.6$ & 30.1 & 28.3 & 29.0 & 30.1 & 29.5 & 29.2 & 29.5 & 30.1 \\
\hline$H$ & $1.67^{[b]}$ & 1.49 & 1.23 & 1.47 & 1.43 & {$[\mathrm{~d}]$} & $1.40^{[\mathrm{e}]}$ & 1.53 & 1.53 \\
\hline C-1 & 48.8 & 48.5 & 48.9 & 51.6 & 49.1 & 50.3 & 50.4 & 48.8 & 47.2 \\
\hline $\mathrm{C}-2$ & 189.1 & 185.1 & 195.6 & 221.6 & 183.8 & 200.0 & 202.2 & 178.2 & 179.1 \\
\hline C-3 & 49.1 & 48.5 & 48.9 & 50.4 & 50.5 & 50.3 & 51.8 & 50.0 & 47.5 \\
\hline C-4 & $122.8^{[\mathrm{f}]}$ & 122.6 & 123.2 & 122.6 & 122.59 & 122.5 & $122.6^{[\mathrm{g}]}$ & 122.5 & 122.5 \\
\hline C-5 & $127.6^{[\mathrm{h}]}$ & 127.7 & 127.2 & 128.3 & 127.5 & 127.8 & $128.0^{[\mathrm{i}]}$ & 128.5 & $127.4^{[\mathrm{k}]}$ \\
\hline$C-6$ & $127.5^{[\mathrm{h}]}$ & 127.7 & 127.2 & 128.3 & 127.5 & 127.8 & $127.8^{[\mathrm{i}]}$ & 128.5 & $127.2^{[\mathrm{k}]}$ \\
\hline $\mathrm{C}-7$ & $122.2^{[\mathrm{f}]}$ & 122.6 & 123.2 & 122.7 & 122.61 & 122.5 & $122.5^{[\mathrm{g}]}$ & 122.1 & 122.5 \\
\hline $\mathrm{C}-8$ & 147.6 & 145.6 & 147.7 & 145.2 & 148.1 & 146.3 & 146.7 & 146.0 & 148.9 \\
\hline C-9 & 146.4 & 145.6 & 147.7 & 144.6 & 147.4 & 146.3 & 144.0 & 144.6 & 147.4 \\
\hline ipso-C & 145.5 & $184.7^{[1]}$ & $1.9^{[\mathrm{m}]}$ & $112.4^{[n]}$ & 140.4 & 146.7 & 140.7 & - & - \\
\hline ortho $-\mathrm{C}$ & 113.1 & $26.3^{[0]}$ & $0.27[\mathrm{p}]$ & - & 123.6 & 125.5 & 126.9 & - & - \\
\hline meta-C & $125.2, \quad 133.3$ & - & - & - & 128.8 & 129.0 & 128.9 & - & - \\
\hline para-C & $122.5^{[q]}$ & - & - & - & 125.4 & 131.2 & 132.9 & $\cdot$ & - \\
\hline
\end{tabular}

[a] In THF/Ether (3:1). - ${ }^{\text {bb] }}$ In $\mathrm{CH}_{2} \mathrm{Cl}_{2}$ - [cl 1.62 und 1.80 bei $-48^{\circ} \mathrm{C}$. - [d] 1.40 und 1.50 bei $-48^{\circ} \mathrm{C}$ - [e] Bei $-47^{\circ} \mathrm{C}$. ${ }^{[\mathrm{f}-\mathrm{k}]} \delta$-Werte mit gleicher Markierung vertauschbar. $-{ }^{\left[{ }^{[]}\right]}$Acetyl-CO. $-{ }^{[\mathrm{m}]} \mathrm{SiMe}_{3} .{ }^{[\mathrm{n}]}$ Cyan. $-{ }^{[0]} \mathrm{Acetyl}^{-\mathrm{CH}_{3} .}-{ }^{[\mathrm{pl}} \mathrm{SiMe}_{3}-\mathrm{Protonen}$ _- ${ }^{\text {[q] }}$ Unsicher; weitere $\mathrm{CH}$ bei $123.4,125.2,126.0$. 
gekoppelter Spektren und gegebenenfalls aus der paarweisen Koaleszenz der Signale konstitutionell äquivalenter CAtome (Abschnitt B). Die sny/anti-Zuordnung der ${ }^{1} \mathrm{H}$ - und ${ }^{13} \mathrm{C}$-Resonanzlinien ist für die folgende NMR-dynamische Untersuchung nicht wesentlich und wurde nur für $\mathbf{3 h}$ gesichert (relativ zur angenommenen ${ }^{1} \mathrm{H}$-Sequenz der Methylgruppen), sonst aber in Analogie zur eindeutigen ${ }^{[1]}$ Zuordnung von 3a vorgenommen (lediglich $3 \mathbf{c}$ analog zu $3 \mathbf{b}$ ).

\section{B. Stereodynamik der Imino-Funktionen}

Zur Geschwindigkeitsmessung der $(E, Z)$-Konfigurationsumwandlung im System 5 eignen sich vorwiegend die NMR-Signalpaare der ${ }^{13} \mathrm{C}$-Kerne C-1/C-3, C-8/C-9 und $1-\mathrm{CH}_{3} / 3-\mathrm{CH}_{3}$ oder der 1,3-Methylprotonen, soweit deren chemische Verschiebungen (Tab. 1) sich bei erhöhten Temperaturen im 1:1-Verhältnis mitteln (koaleszieren). Die Geschwindigkeitskonstanten $k(T)$ ergeben sich daher durch Linienformanalysen für den Austausch ungekoppelter Spinsysteme ${ }^{[18,19]}$ oder bei den jeweiligen Koaleszenztemperaturen $T_{\mathrm{c}}$ durch die Beziehung $k\left(T_{\mathrm{c}}\right)=\Delta v \cdot \pi / \sqrt{2}$ mit dem Frequenzabstand $\Delta v$ im koaleszierenden Signalpaar. Die daraus berechneten freien Aktivierungsenthalpien $\Delta G^{*}$ sind unabhängig von der Temperatur, wenn die Aktivierungsentropien $\Delta S^{*}$ null sind; die Fehlerangaben in Tab. $2 \mathrm{zu}$ diesen Aktivierungsparametern berücksichtigen auch die Temperatur-Meßfehler ${ }^{[19]}$.

Bei $N$-Alkyliminen wie $\mathbf{6 a}$ ist die $(E, Z)$-Konfigurationsumwandlung so langsam, $\mathrm{da} B$ sie konventionell verfolgt ${ }^{[8,20.21]}$ und nur bei den höchsten erreichbaren Temperaturen auf die NMR-Zeitskala gebracht werden $\operatorname{kann}^{[21-23]}$. Da nur monomolekulare Mechanismen ${ }^{[2]}$ eine direkte Interpretation der Stereodynamik erlauben, muß man die Möglichkeit konkurrierender bimolekularer Prozesse mit pseudo-erster Reaktionsordnung bei höheren Temperaturen besonders beachten. Wenn das Imin azide $\alpha$-Protonen wie in 7 besitzt, kann sich das sec-Enamin als Zwischenstufe bilden, über dessen wenig behinderte CN-Rotation dann die indirekte Konfigurationsumwandlung erfolgt. Diese Tautomerisierung kann sehr mobil sein ${ }^{[22]}$ und sogar - erkannt oder unerkannt - die $(E, Z)$-Gleichgewichtseinstellung der sonst notorisch konfigurationsstabilen Hydrazone beeinflussen ${ }^{[25-27]}$. Da dieser Mechanismus in den Systemen 5 und $\mathbf{6}$ ausgeschlossen ist, vergleichen wir $\mathbf{3 a}$ mit $\mathbf{6}^{[8,20,22,23]}$ (statt mit 7a) und schließen daraus in Tab. 2 auf eine sterisch bedingte Beschleunigung $\Delta \Delta G^{*}=-2.9 \mathrm{kcal} / \mathrm{mol}$. Die etwas höhere Barriere $\Delta G^{\neq}=23.9 \mathrm{kcal} / \mathrm{mol}$ des Bis- $N$ -

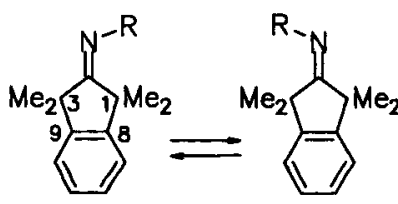

5: $3 \mathbf{a}-\mathbf{c}, \mathbf{e}, \mathbf{h}-\mathbf{k}$<smiles>[R]N=C([Y])[Y]</smiles>

6: $\mathrm{X}, \mathrm{Y}=$ Arylreste

7: $\mathrm{X}=\mathrm{Y}=\mathrm{CH}_{3}$<smiles>[R]N=C([Y6])C([Y])=[V]</smiles>

8: $\mathrm{X}=\mathrm{NR}$

9: $\mathrm{X}=0$

\begin{tabular}{c|c} 
6- & $\mathbf{R}$ \\
\hline $\mathbf{a}$ & $\mathrm{Me}$ \\
$\mathbf{b}$ & $\mathrm{Ph}$ \\
$\mathbf{e}$ & $\mathrm{CO}^{-\mathrm{CH}_{3}}$ \\
$\mathbf{g}$ & $\mathrm{SiMe}_{3}$ \\
$\mathbf{h}$ & $\mathrm{CN}$ \\
$\mathbf{i}$ & $\mathrm{S}-\mathrm{Ph}$ \\
$\mathbf{j}$ & $\mathrm{SO}^{-\mathrm{Ph}}$ \\
$\mathbf{k}$ & $\mathrm{SO}_{2}-\mathrm{Ph}$ \\
$\mathbf{m}$ & $\mathrm{CMe}_{3}$
\end{tabular}

Tab. 2. Freie Aktivierungsenthalpien $\Delta G^{\neq}$und $\Delta \Delta G^{\neq}$(kcal $/ \mathrm{mol}$ ) sowie Aktivierungsentropien $\Delta S^{\ddagger}$ (cal mol ${ }^{-1} \mathrm{~K}^{-1}$ ) der Imine 3 mit Vergleichsdaten ${ }^{\text {[Lit.] }}$ von 6 und 7

\begin{tabular}{|c|c|c|c|c|c|c|c|}
\hline \multirow{2}{*}{$\frac{\mathrm{R}}{\mathrm{CH}_{3}}$} & \multirow[t]{2}{*}{3} & \multicolumn{2}{|c|}{$\Delta G^{\#}$} & \multirow{2}{*}{$\begin{array}{l}\text { Solvens, } \\
\text { Anisol, }\end{array}$} & ${ }^{\circ} \mathrm{C}$ & $\Delta S^{\#}$ & \multirow{2}{*}{$\frac{\Delta \Delta G^{\#}}{-2.9}$} \\
\hline & & 23.0 & $( \pm 0.1)$ & & 147 & ca. 0 & \\
\hline $\mathrm{C}_{6} \mathrm{H}_{5}$ & b & 15.40 & $( \pm 0.01)$ & Anisol, & 25 & $-1.4( \pm 2)$ & -2.7 \\
\hline 1-Naph & c & 14.50 & $( \pm 0.02)$ & Anisol $[\mathrm{a}]$ & 25 & $-1.7( \pm 2)$ & $-3.6^{[b]}$ \\
\hline $\mathrm{COCH}_{3}$ & e & 8.3 & $( \pm 0.1)$ & $\mathrm{CDCl}_{3}$ & -85 & - & $>-4$ \\
\hline $\mathrm{CN}$ & h & 15.35 & $( \pm 0.01)$ & $\mathrm{CDCl}_{3}$, & 25 & $0 \quad( \pm 2)$ & $>-3.5$ \\
\hline$S-\mathrm{C}_{6} \mathrm{H}_{5}$ & i & 18.34 & $( \pm 0.05)$ & $\mathrm{Ph}_{2} \mathrm{O}$ & 72 & $0 \quad( \pm 2)$ & -0.2 \\
\hline SO-Ph & $j$ & 12.85 & $( \pm 0.01)$ & $\mathrm{CDCl}_{3}$, & -24 & $-0.7( \pm 2)$ & -1.2 \\
\hline $\mathrm{SO}_{2}-\mathrm{Ph}$ & $\mathbf{k}$ & 11.5 & $( \pm 0.1)$ & $\mathrm{CDCl}_{3}$, & -42 & $0 \quad( \pm 7)$ & -1.9 \\
\hline $\mathbf{R}$ & 6 & $\Delta G^{\#}$ & Solven & [Lit] & 7 & $\Delta G^{\#}$ & Solvens ${ }^{[L i t]}$ \\
\hline $\mathrm{CH}_{3}$ & a & $26.0 ; 25.7$ & $\mathrm{C}_{6} \mathrm{H}_{12}$ [c & 3]; $\quad \mathrm{Ph}_{2}[\mathrm{c}, 22]$ & $\mathbf{a}$ & - & - \\
\hline $\mathrm{C}_{6} \mathrm{H}_{5}$ & b & 18.1 & $\mathrm{CCl}_{4}^{[36]}$ & & b & 20.3 & $\mathrm{Ph}_{2} \mathrm{O}^{[37]}$ \\
\hline $\mathrm{COCH}_{3}$ & e & $<13$ & Aceton $[3$ & & e & - & - \\
\hline $\mathrm{CN}$ & h & - & - & & h & 18.9 & Aceton $[54]$ \\
\hline $\mathrm{S}-\mathrm{C}_{6} \mathrm{H}_{5}$ & $\mathbf{i}$ & $18.6 ; 18.4$ & $\mathrm{CDCl}_{3}{ }^{5}$ & -61]; $\mathrm{DCB}^{[\mathrm{d}]}$ & i & 19.3 & $\mathrm{CDCl}_{3}{ }^{[61]}$ \\
\hline SO-Ph & $\mathbf{j}$ & 14.1 & $\mathrm{DCB}^{[\mathrm{d}]}$ & & $\mathbf{j}$ & 17.0 & - [64] \\
\hline $\mathrm{SO}_{2}-\mathrm{Ph}$ & $\mathbf{k}$ & $13.5 ; 13.0$ & $\mathrm{CDCl}_{3}[6$ & ; $\mathrm{DCB}^{[\mathrm{d}]}$ & k & $16.3^{[e]}$ & $\mathrm{CH}_{2} \mathrm{Cl}_{2}{ }^{[65]}$ \\
\hline
\end{tabular}

[a] Auch in $\mathrm{CDCl}_{3}$. - ${ }^{[b]}$ Relativ zu 6b. $-{ }^{[c]}$ Auch in Diphenylether ${ }^{[20,23]}$. - ${ }^{[\mathrm{d}]}$ In $o$-Dichlorbenzol ${ }^{[59]}$. - ${ }^{[e]} N$-(Phenylsulfonyl)imin von 3,4-Dimethyl-3-penten-2-on ${ }^{[65]}$.

methylimins $8 \mathbf{a}$ in Trichlorbenzol ${ }^{[28]}$ schreiben wir also dessen geringerer Vorderseitenspannung ${ }^{[29]} \mathrm{zu}$.

Ein weiterer bimolekularer Mechanismus der Konfigurationsumwandlung besteht in der Additions-Eliminierungsreaktion bei der Einwirkung nucleophiler Katalysatoren wie Benzoesäure ${ }^{[23]}$ und Anilin ${ }^{[30]}$ oder in der Rückseiten-Solvatation ${ }^{[31]}$; er kann auch bei 6 vorkommen ${ }^{[23]}$. Für das System 5 ist er aber wenig wahrscheinlich: Da alle Imine 3a-e und 3h-I sehr langsam oder gar nicht hydrolysiert werden, muß der nucleophile Angriff am sterisch abgeschirmten Zentrum C-2 sehr behindert sein. Die Bildung des Hydrazons ${ }^{[32]}$ hatte ebenfalls lange Reaktionszeiten bei hohen Temperaturen erfordert.

Um bimolekulare Mechanismen auch direkt auszuschlieBen und zugleich den Raumbedarf bei der Stereomutation zu prüfen, haben wir am Beispiel 3a das Aktivierungsvolumen bestimmt, das für Reaktionen pseudo-erster Ordnung deutlich negativ sein sollte ${ }^{[33]}$. Bis 1500 bar steigt die Koaleszenztemperatur in 1,2-Dibrom-tetrafluorethan von ca. $+188(300 \mathrm{MHz})$ auf $194^{\circ} \mathrm{C}$. Da die Geschwindigkeitskonstanten $k$ sehr genau bestimmt werden müssen, weil ihre Druckabhängigkeit bei so hohen Temperaturen schon recht gering ist, haben wir diese ${ }^{1} \mathrm{H}$-NMR-Linienformmessungen bei variabler Temperatur vorgenommen und das Aktivie- 
rungsvolumen $\Delta V^{\neq}=+10.2( \pm 1.5) \mathrm{cm}^{3} \mathrm{~mol}^{-1}$ aus den $k$-Werten bestimmt, die bei $+190^{\circ} \mathrm{C}$ im Arrhenius-Diagramm interpoliert wurden. Abb. 1 zeigt auch, daß beim Solvenswechsel zum polareren Anisol (bei $60 \mathrm{MHz}$ ) fast keine Änderung eintritt; im $\Delta G^{\ddagger}$-Wert (Tab. 2) drückt sich der geringe Unterschied nicht mehr aus. Über beide Lösungsmittel gemeinsam berechnet wird die Aktivierungsentropie etwa null, also ähnlich wie z. B. für $\mathbf{6 a}^{[20]}$.

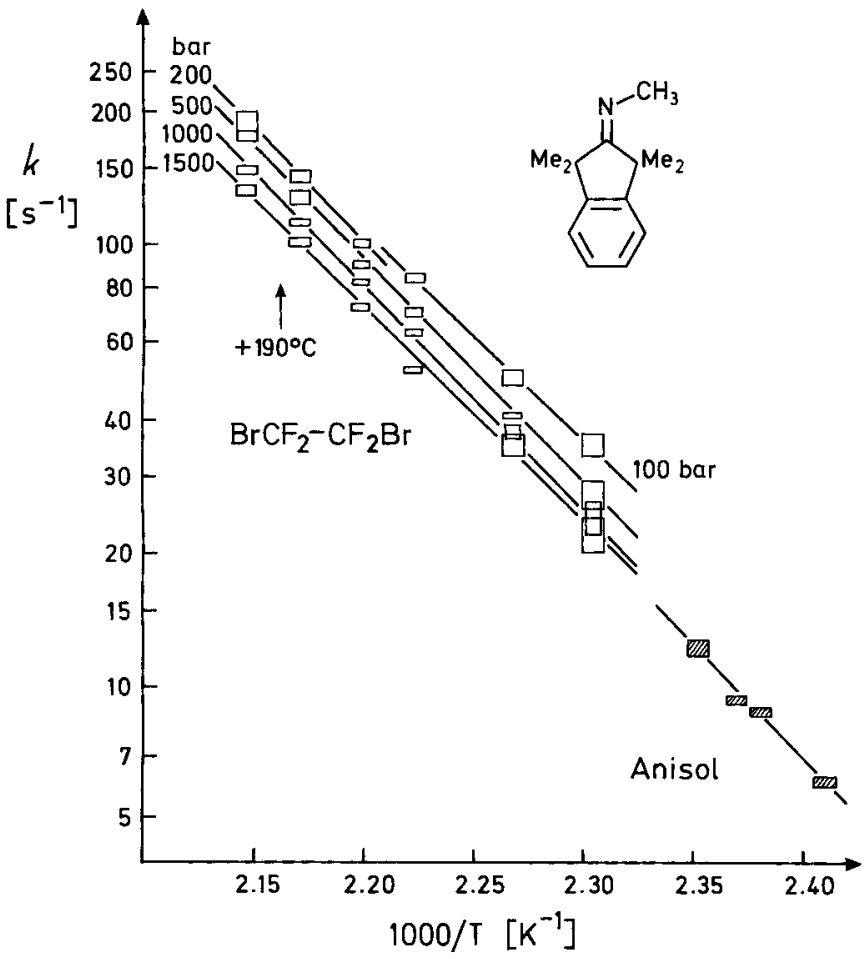

Abb. 1. Arrhenius-Diagramm der $(E, Z)$-Stereomutations-Geschwindigkeitskonstanten $k$ von 3a bei unterschiedlichen Drucken in 1,2Dibrom-1,1,2,2-tetrafluorethan; schraffierte Meßpunkte in Anisol bei Normaldruck

Der für 3a gefundene $\Delta V^{*}$-Wert erscheint also zuverlässig und übertrifft noch diejenigen von tertiären bicyclischen (Z)-Azoalkanen ${ }^{[34,35]}$. Stark positive Aktivierungsvolumina können bedeuten, daß der molekulare Übergangszustand mehr Platz beansprucht als der Grundzustand oder weniger polar ist (Elektrorelaxation ${ }^{[33]}$ des Lösungsmittels). Die für 3a zu vermutende ${ }^{[9]}$ sterische Pressung ${ }^{[33]}$ könnte in gleicher Richtung wirken; zwar können wir diese Faktoren nicht separieren, doch läßt die Größe des beobachteten $\Delta V^{+}$nur sehr wenig Spielraum für negative Beiträge durch erhöhte Polarität des Übergangszustands oder bimolekulare $\mathrm{Me}$ chanismen.

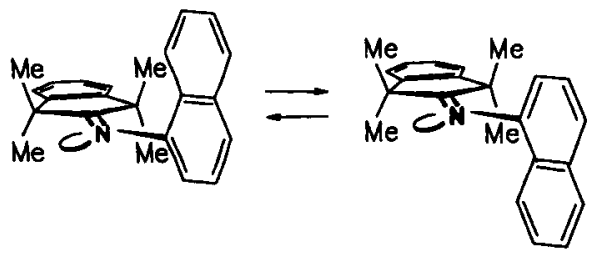

$10(=3 c) \quad 11_{(=3 c)}$
Die $N$-Phenylverbindung $3 \mathrm{~b}$ ist um fast $3 \mathrm{bzw} .5 \mathrm{kcal} / \mathrm{mol}$ konfigurationslabiler (Tab. 2) als die Modellsysteme $\mathbf{6 b}^{[36]}$ und $\mathbf{7} \mathbf{b}^{[37]}$. Sowohl bei $\mathbf{9} \mathbf{b}^{[37,38]}$ mit geringerer Vorderseitenspannung ${ }^{29]}$ als auch bei $6 \mathbf{b}$ mit $o$-Tolyl- oder FluorenylSubstituenten ${ }^{[39,40]}$ fehlt dieser beschleunigende Effekt.

Die Gerüstebenen der $N$-Arylgruppen in $\mathbf{3 b}$ und erst recht in 3c $(=10)$ stehen zweifellos orthogonal zur CN-Doppelbindungsebene, wie durch die verwandten Strukturen eines Imins $^{[9]}$ und des mit $\mathbf{3 b}$ isoelektronischen 2-Benzyliden1,1,3,3-tetramethylindans ${ }^{[41]}$ nahegelegt wird. Das $N-(1$ Naphthyl)imin 3c ist somit chiral und seine $(E, Z)$-Konfigurationsumwandlung eine Enantiomerisierung, die laut Tab. 2 gegenüber 3b noch einmal beschleunigt ist. Der Temperaturgang der den Aktivierungsparametern von $3 \mathbf{c} z \mathfrak{u}-$ grunde liegenden $k$-Werte ist in Abb. 2 gezeigt, um die hier vollkommene Übereinstimmung in den beiden Lösungsmitteln Anisol und Chloroform zu belegen. Unterhalb von $-10^{\circ} \mathrm{C}$ friert auch noch die $\mathrm{CN}$-Einfachbindungs-Rotation $(\mathbf{1 0} \leftrightarrows 11)$ auf der NMR-Zeitskala ein, was sich durch vier gleichgroße NMR-Signale für die vier Methylgruppen in 10 verrät (Exp. Teil). Diese Rotation bedeutet ebenfalls Enantiomerisierung; sie erfordert in $\mathrm{CH}_{2} \mathrm{Cl}_{2}$-Lösung $\Delta G^{\neq}=$ca. $11.3 \mathrm{kcal} / \mathrm{mol}$ und in $\mathrm{CDCl}_{3} 10.7(1) \mathrm{kcal} / \mathrm{mol}\left({ }^{13} \mathrm{C}\right.$-Linienformanalyse). Bei C-(1-Naphthyl)-Rotationen der Imine von $\alpha$-Naphthylketonen sind dagegen höhere Energieschwellen gemessen worden ${ }^{[42-44]}$.

Die $N$-Acetylgruppe in 3e ist als $\pi$-Akzeptor prädestiniert, das freie Elektronenpaar des Stickstoffs in einem linearen Übergangszustand 13 durch Mesomerie zu stabilisieren. In der Tat läßt sich der sehr kleine $\Delta G^{*}$-Wert 8.3(1) kcal/mol

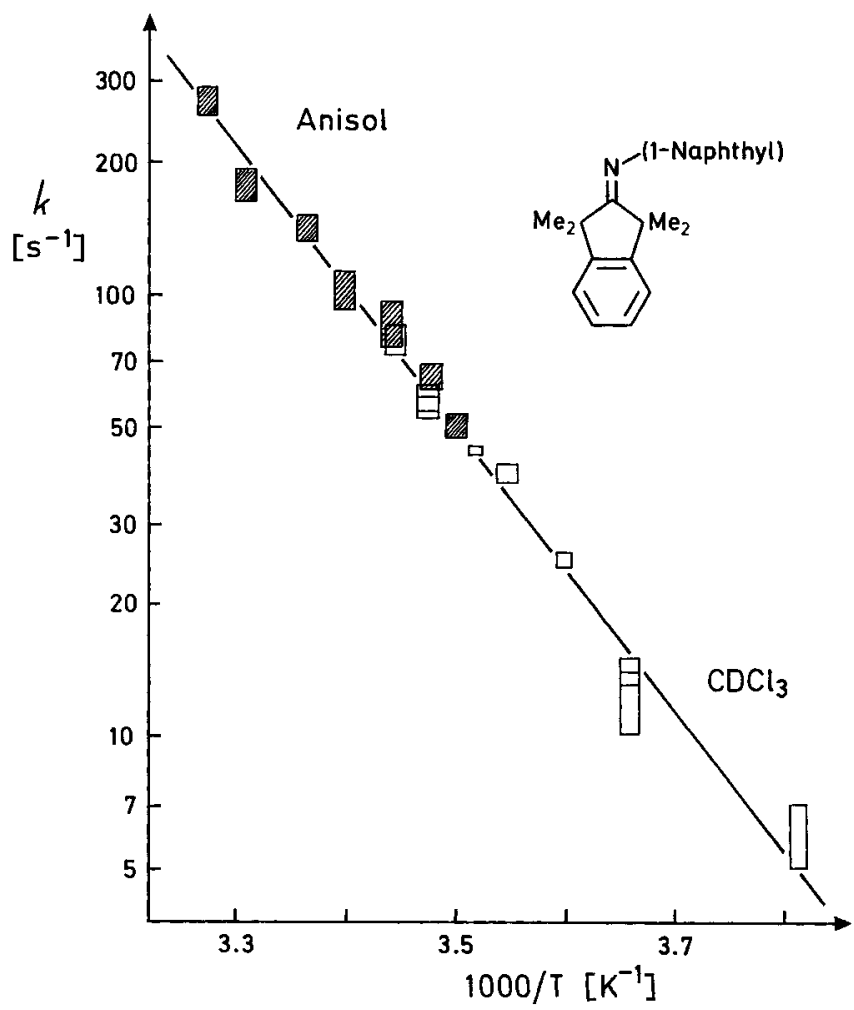

Abb. 2. Arrhenius-Diagramm der (E,Z)-Stereomutations-Geschwindigkeitskonstanten $k$ von $3 \mathrm{c}$ in Anisol (schraffiert) und in $\mathrm{CDCl}_{3}$ 
(Tab. 2) nur wegen der großen ${ }^{13} \mathrm{C}$-Verschiebungsdifferenz der 1,3-Methylgruppen (Tab. 1) feststellen, die als einziges diastereotopes Paar erst unterhalb von $-75^{\circ} \mathrm{C}(100.6 \mathrm{MHz})$ eine Signalaufspaltung zeigen. Hierdurch und mit der sehr niedrig berechneten ${ }^{\left[{ }^{[3]}\right.}$ Energieschwelle klärt sich, weshalb an anderen $N$-Acyliminen ${ }^{[11-13,37]}$ wie 6e keine Aufspaltung gefunden werden konnte $\left(<13 \mathrm{kcal} / \mathrm{mol}^{[37]}\right)$. Als obere Schranke für ein sterisch nicht beschleunigtes $N$-Acetylimin 6e kann man $\Delta G^{*}=12.5 \mathrm{kcal} / \mathrm{mol}$ des entsprechenden Carbaminats ${ }^{[45]}$ ansehen. Die bekannten Strukturen dieser beiden Referenzverbindungen ${ }^{[13,45]}$ zeigen, daß sogar in den gewinkelten Grundzuständen die Konjugation der $N$-Acylgruppen mit dem freien Elektronenpaar bevorzugt wird.

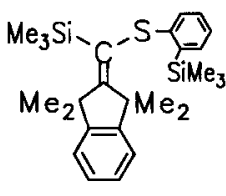

12

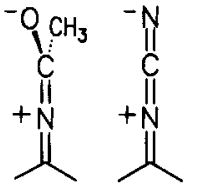

13

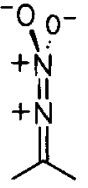

15

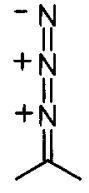

16
Die $N$-Trimethylsilylverbindung $\mathbf{3 g}$ zeigt dagegen bis $-70^{\circ} \mathrm{C}$ überhaupt keine diastereotope Aufspaltung. Nach Berichten über einige $N$-Silylimine ${ }^{[46-48]}$ wie $6 \mathrm{~g}$ (oder sterisch überladene $\left.{ }^{[49]}\right)$ ist dies nicht überraschend. Auch die starke Winkeldeformation in der Struktur ${ }^{[50]}$ des analogen Olefins 12 legt es nahe, eine große Beschleunigung durch Vorderseitenspannung zu erwarten; mit $N$-ständigen tertButylgruppen ${ }^{[20,22,28,51,52]}$ beträgt sie schon ca. $-5.5 \mathrm{kcal} / \mathrm{mol}$ bei $\mathbf{6} \mathbf{m}^{[20,22]}$ (bezogen auf 6a). Durch Ablösung des Silylrestes vom Stickstoff ${ }^{\left[{ }^{33]}\right.} \mathrm{kann}$ bei $\mathbf{3 g}$ die $(E, Z)$-Stereomutation nicht vorgetäuscht sein, weil $\mathbf{3 g}$ und $\mathbf{3 f}$ in Koexistenz noch bei $+25^{\circ} \mathrm{C}$ separate NMR-Signale ergeben.

Daß der $N$-Cyan-Substituent in $7 \mathbf{h}^{[54]}$ die $(E, Z)$-Konfigurationslabilität noch etwas mehr als die $N$-Phenylgruppe in $\mathbf{7} \mathbf{b}^{[37]}$ erhöht, trifft auch für $\mathbf{3 h}$ zu (Vergleich mit $\mathbf{3 b}$ in Tab. 2). Bei ${ }^{1} \mathrm{H}-\mathrm{NMR}-$ Koaleszenztemperaturen um $+28^{\circ} \mathrm{C}$ $(80 \mathrm{MHz})$ fällt die größere $(E, Z)$-Topomerisierungsrate im polareren $\mathrm{CDCl}_{3}$ gegenüber $\mathrm{CCl}_{4}$ zwar besonders auf, doch entspricht sie nur einer 2-3fachen Beschleunigung; sollte 14 den Übergangszustand beschreiben ${ }^{[55]}$, so ist dieser also kaum polarer als der Grundzustand. Gegenüber $7 \mathrm{~h}$ beträgt die sterisch verursachte Beschleunigung $-3.5 \mathrm{kcal} / \mathrm{mol}$ (Tab. 2) und liegt damit ähnlich wie bei $N$-Cyanchinoniminen ${ }^{[56]}$, in deren Struktur ${ }^{[57]}$ ebenfalls Vorderseitenspannung erkennbar ist.

Die Fähigkeit der Schwefelfunktionen in $\mathbf{3 i}-\mathbf{k}$, auch die $(E, Z)$-Stereomutation der Iminogruppe zu erleichtern, ist schon lange bekannt ${ }^{[58]}$. Im $N$-Phenylthio-Derivat 3i ist die sterisch bedingte Beschleunigung auffallend gering (Tab. 2), wenn man mit $6 \mathbf{i}^{[59-61]}$ oder $7 \mathbf{i}^{[61]}$ vergleicht. Dem entspricht der nicht aufgeweitete Winkel ${ }^{[50]} 117.5(2)^{\circ}(\mathrm{C}=\mathrm{C}-\mathrm{S})$ im analogen Olefin 12. Die überraschend niedrige Barriere (13.7 $\mathrm{kcal} / \mathrm{mol}^{\left[{ }^{62}\right)}$ ) eines $N$-(Methylthio)chinon-imins ist also möglicherweise durch eine unerkannte bimolekulare Katalyse vorgetäuscht worden, doch lassen sich in dessen Struktur ${ }^{[62]}$ auch Anzeichen von Vorderseitenspannung erkennen. Wie bei $\mathbf{6 i}^{\left[\mathbf{i}^{161}\right.}$ ist auch bei $3 \mathbf{i}$ die Aktivierungsentropie effektiv null.

Wegen seiner „Sulfoxid“-Funktion bleibt das $N$-Phenylsulfinyl-Derivat $\mathbf{3 j}$ im Unterschied zu $\mathbf{3 c}$ auch bei rascher $(E, Z)$-Topomerisierung chiral und nimmt dabei effektive $C_{2}$ Symmetrie an, so da 3 man immer mindestens zwei Protonensorten für die vier Methylgruppen findet. Die bei $-48^{\circ} \mathrm{C}$ beobachteten vier Methylgruppensignale sind alle gleich intensiv, und die beiden inneren Komponenten $(\delta=1.50$ und 1.62) koaleszieren separat von den beiden äußeren $(\delta=1.40$ und 1.80). Wegen der obigen $C_{2}$-Symmetrie bedeutet dies, daß trans-ständige 1,3-Methylgruppen ihre Plätze tauschen. Die kürzlich publizierte ${ }^{[63]}$ Struktur eines Acetophenon-sulfinylimins ist bei der genaueren Zuordnung weniger hilfreich als das Methylprotonenspektrum der $N$-PhenylsulfonylVerbindung 3k, das nur die beiden äußeren Komponenten der Vierergruppe von 3j aufweist. Deren $\delta$-Werte (Tab. 1) werden also vom Einfluß der $\mathrm{SO}_{2}$ - bzw. SO-Sauerstoffatome geprägt, die der inneren Komponenten dagegen vom freien Elektronenpaar des Sulfoxid-Schwefels, so da $\beta$ sie denen von $3 i$ gleichen.

Die sterisch bedingte Beschleunigung $(-1.2 \mathrm{kcal} / \mathrm{mol})$ von $3 \mathbf{j}$ gegenüber $\mathbf{6} \mathbf{j}^{[59]}$ (anstelle von $\mathbf{7} \mathbf{j}^{[64)}$ ) ist nicht groß, wächst aber etwa additiv mit der Einführung des zweiten Sauerstoffatoms in $3 \mathbf{k}$ auf $-1.9 \mathrm{kcal} / \mathrm{mol}$ an (Vergleich mit $\mathbf{6} \mathbf{k}^{[59,60]}$ anstelle von $7 \mathbf{k}^{[55)}$ ). Der Gang der $\Delta G^{\neq}$-Werte von $3 \mathbf{a}, \mathbf{b}, \mathbf{h}$ und $\mathbf{k}$ ist auch demjenigen bei Imino-dithiolcarbonaten ${ }^{[66]}$ verwandt.

An $N$-Nitroiminen wurde nie $(E, Z)$-Koaleszenz beobachtet ${ }^{[67-70]}$. Trotz der erwarteten Vorderseitenspannung zeigt auch $3 \mathrm{I}^{[10]}$ bis $+170^{\circ} \mathrm{C}$ scharfe NMR-Signale (also $\Delta G^{\neq} \gg 24.3 \mathrm{kcal} / \mathrm{mol}$ ) und zersetzt sich dann zu 1,1,3,3Tetramethyl-2-indanon. In entsprechender Weise zerfallen auch sterisch unbelastete Nitrimine ${ }^{[71]}$ noch leichter als belastete $^{[67]}$. Wie beim Nitrimin des 4-Nitrocamphers ${ }^{[2]}$ sollte sich die Nitrogruppe schon im Grundzustand von 31 etwa orthogonal zur CN-Doppelbindungsebene einstellen. Diese Konformation wäre auch in einem linearen Übergangszustand 15 für die Delokalisation des freien Elektronenpaares geeignet; im Gegensatz zu 13 und 14 verhindert die Nachbarschaft der beiden positiven Formalladungen in 15 jedoch wie bei den Nitraminen ${ }^{[7]}$ eine wirksame Mesomeriestabilisierung. Dieser Effekt dürfte auch die hohe Barriere von 16 (ca. $28.2 \mathrm{kcal} / \mathrm{mol}^{[55]}$ ) mit der als $\pi$-Akzeptor noch stärkeren ${ }^{[74]}$ Diazoniumgruppe erklären. Konsequenterweise zeigen die phenylog gebundenen Acetyl-, Cyan-, Nitro- und Diazonium-Gruppen jedoch ihre normalen Substituenteneinflüsse ${ }^{[75]}$.

\section{Zusammenfassung und Schlubfolgerungen}

Das Kohlenstoffatom der CN-Doppelbindung in 5 wird von den tertiären Alkylsubstituenten gegen nucleophile Reagentien stark abgeschirmt. Am $\mathrm{sp}^{2}$-Stickstoffatom ist die Reaktivität gegenüber Chlortrimethylsilan und Acetylchlorid deutlich verringert, die Protonierung aber erst mit dem $N$-(1-Naphthyl)-Substituenten in 3c sterisch behindert. Auch die nicht mehr basischen Imine $\mathbf{3 e}, \mathbf{j}$ und $\mathbf{k}$ werden durch 
wäßrige Säure langsam hydrolysiert, also wohl nach der $N$-Protonierung ebenfalls nur schwer am Kohlenstoffatom angegriffen. Bimolekulare Mechanismen der $(E, Z)$-Stereomutation sind deswegen unwahrscheinlich, bei höheren Temperaturen allerdings nicht grundsätzlich auszuschlieBen. Aber sogar im Fall 3a spricht das positive Aktivierungsvolumen gegen bimolekulare Katalyse durch unerkannte Verunreinigungen. $\mathrm{Da} \beta$ die $(E, Z)$-Stereomutation also monomolekular abläuft, ist auch mit den überall sehr kleinen Beträgen der Aktivierungsentropien vereinbar.

Aus diesen Gründen und wegen ihrer meistens sehr geringen konformativen Flexibilität eignen sich die vorgestellten Verbindungen zur Eichung von Kraftfeldparametern für die CN-Doppelbindung, bei denen insbesondere das Problem der Stickstoff-Inversion rechnerisch noch nicht befriedigend gelöst erscheint ${ }^{[76]}$. Eine erwünschte Erweiterung ${ }^{[76]}$ der experimentellen Basis dafür bieten die $\Delta G^{+}$-Werte (Tab. 2) der (E,Z)-Stereomutation, weil sie mit den Aktivierungsenthalpien $\Delta H^{\ddagger}$ praktisch identisch sind. Die von uns versuchte Quantifizierung der Vorderseitenspannung $\left(\Delta \Delta G^{\mp}\right.$ in Tab. 2) drückt dies in anderer Weise aus und sollte sich auf die analoge $(E, Z)$-Topomerisierung der isoelektronischen Vinyllithiumverbindungen 2 anwenden lassen.

Ein klares Beispiel für den Einfluß der Vorderseitenspannung auf die Ethanolysegeschwindigkeit von Sulfonsäureestern haben Takeuchi et al. ${ }^{177]}$ soeben beschrieben und dabei auf die Notwendigkeit hingewiesen, starre Modellsysteme zu verwenden. Die dort gefundene Beschleunigung um $-3 \mathrm{kcal} / \mathrm{mol}^{[77]}$ durch eine syn-Methylgruppe entspricht dem für 3a in Tab. 2 angegebenen Wert.

Der Mechanismus der monomolekularen $(E, Z)$-Stereomutation kann an der chiralen $N$-(1-Naphthyl)-Verbindung $3 c$ noch nicht ermittelt werden. Denn die enantiomerisierende Rotation der 1-Naphthylgruppe um die CN-Einfachbindung ist der schnellste beobachtete Vorgang; er müßte durch weitere Substituenten verlangsamt werden, was an synthetischen Schwierigkeiten scheiterte.

Wegen der mangelnden Lösungsmittelabhängigkeit der Stereomutation wäre auch die Quantifizierung der elektronischen Substituenteneinflüsse nach Hammett durch $p$-substituierte Derivate von 3b von Interesse. Aus synthetischen Gründen läßt sich dies aber zweckmäßiger bei sterisch noch stärker abgeschirmten Analoga ${ }^{[75]}$ durchführen.

Substituenten-induzierte chemische Verschiebungen (SCS) können leicht aus Tab. 1 durch Subtraktion der ${ }^{13} \mathrm{C}$ Daten ${ }^{[1]}$ des Imins 3d berechnet werden. Da die Zuordungen zwar wahrscheinlich, aber nur für $\mathbf{3 h}$ auf Relativbasis gesichert sind, werden die SCS-Werte hier nicht explizit angegeben; sie ähneln denjenigen von später zu publizierenden, isoelektronischen Olefinderivaten. Sicher erkennbar ist jedoch auch hier die Tendenz zur Polarisation des Benzolringes ${ }^{[1]}$, die an C-8 und C-9 entweder zur Hochfeld- oder zur Tieffeldverschiebung (relativ zu $\delta=147.1$ für $\mathbf{3 d}^{[1]}$ ) führt, wenn die $N$-Substituenten als elektronische Akzeptoren bzw. Donoren wirken.

Der Deutschen Forschungsgemeinschaft und dem Fonds der Chemischen Industrie danken wir für ihre großzügige Hilfe.

\section{Experimenteller Teil}

IR: Perkin-Elmer 125 oder Bruker IFS-45. - UV/Vis: PRQ 20 und DMR-10 (C. Zeiss). - MS: Finnigan MAT 90. - NMR: Varian VXR-400S, XL-100-IL und A-60 sowie Bruker WP-80-CW, WP-80-DS und MSL-300, interner Standard TMS.

${ }^{1} \mathrm{H}$ - und ${ }^{13} \mathrm{C}-\mathrm{NMR}$-Linienformanalysen wurden durch Vergleich mit Computer-simulierten ${ }^{[18]}$ Spektren vorgenommen. Die Frequenzabstände $\Delta v$ wurden aus Aufnahmen bei tieferen Temperaturen auf die MeBtemperaturen (Unsicherheit meistens max. $\pm 1 \mathrm{~K}$ ) extrapoliert. Zur Ermittlung der Aktivierungsparameter ${ }^{[19]}$ verwendete man nur solche Spektren, in denen die Halbwertsbreiten der koaleszierenden Banden wenigstens 5mal größer waren ais diejenige eines am Austausch nicht beteiligten Standardsignals. Da die Aktivierungsentropien im Rahmen der Fehlergrenzen nahe bei null lagen, wurde dies auch für Substanzen mit nur einem auswertbaren Koaleszenzpunkt angenommen, was bei Iminen meistens gerechtfertigt ist.

Zur $^{1}$ H-NMR-Koaleszenzmessung unter Druck ${ }^{[78]}$ (Bruker MSL300 bei $300.13 \mathrm{MHz})$ wurde 1,2-Dibrom-1,1,2,2-tetrafluorethan $\left({ }^{19} \mathrm{~F}\right.$ Lock) mit $\mathrm{CaO}$ und aktiviertem $\mathrm{Al}_{2} \mathrm{O}_{3}$ behandelt und von Molekularsieb $3 \AA$ abdestilliert.

$N-(1,1,3,3-T e t r a m e t h y l-2-i n d a n y l i d e n)$ methylamin $(\mathbf{3 a})^{[1]}: 20 \mathrm{mg}$ $3 \mathrm{a}$ in $1.0 \mathrm{~g}$ 1,2-Dibrom-1,1,2,2-tetrafluoroethan wurden bei Drukken bis zu 1500 bar untersucht und die ${ }^{1} \mathrm{H}-\mathrm{NMR}$-Linienformen zwischen 161 und $193^{\circ} \mathrm{C}$ ausgewertet (vgl. Abb. 1). In diesem Lösungsmittel ist der Abstand der gemessenen 1,3-Methylprotonensignale druck- und temperaturabhängig mit $\Delta \delta=0.2190-p$. $5.67 \cdot 10^{-6}\left(\mathrm{ppm} \cdot \mathrm{bar}^{-1}\right)+(T-373 \mathrm{~K}) \cdot 3.3 \cdot 10^{-5}\left(\mathrm{ppm} \cdot \mathrm{K}^{-1}\right)$. Auch in Anisol wuchs der Abstand beim Erhitzen unter Normaldruck mit $\Delta \delta=0.00057 \cdot(T-289 \mathrm{~K})\left(\mathrm{ppm} \cdot \mathrm{K}^{-1}\right)$, also Singulett bei $16^{\circ} \mathrm{C}$, während er in Chinolin umgekehrt bis $+130^{\circ} \mathrm{C}$ auf null schrumpfte.

$N-(1,1,3,3-$ Tetramethyl-2-indanyliden $)$ anilin $(3 \mathbf{b})\left[{ }^{[1]}:{ }^{1} \mathrm{H}-\mathrm{NMR}-\mathrm{Li}-\right.$ nienformanalyse $(60 \mathrm{MHz})$ der Methylgruppen in Anisol zwischen +12.5 und $+43.5^{\circ} \mathrm{C}, T_{\mathrm{c}}=28^{\circ} \mathrm{C}, \Delta \delta=0.283 \mathrm{ppm}$ temperaturkonstant.

1-[(1,1,3,3-Tetramethyl-2-indanyliden) amino]naphthalin $\quad$ (3c): Das für $3 \mathrm{~b}$ angegebene Verfahren ${ }^{[1]}$ ergab in $2 \mathrm{~h}$ bei $190^{\circ} \mathrm{C}$ nur $13 \%$ 3c. Daher ließ man zu $1.43 \mathrm{~g} \mathrm{(10} \mathrm{mmol)} \alpha$-Naphthylamin in $10 \mathrm{ml}$ Mesitylen unter Eiskühlung und Rühren (Argonschutz) langsam $3.95 \mathrm{ml} n$-Butyllithium (2.53 $\mathrm{N}$ in Hexan) tropfen und kühlte die gelbe Suspension auf $-78^{\circ} \mathrm{C}$. Beim Zutropfen von $0.280 \mathrm{ml}(2.5$ mmol) $\mathrm{TiCl}_{4}$ in $2 \mathrm{ml} \mathrm{Mesitylen} \mathrm{wurde} \mathrm{die} \mathrm{Lösung} \mathrm{schwarz.} \mathrm{Man}$ ließ auf Raumtemp. aufwärmen und tropfte eine Lösung von $950 \mathrm{mg}(5 \mathrm{mmol}) 1,1,3,3-$ Tetramethyl-2-indanon ${ }^{[32]}$ in $5 \mathrm{ml} \mathrm{Mesi-}$ tylen $\mathrm{zu}$, destillierte das Hexan vollständig ab und erhitzte $17 \mathrm{~h}$ auf $190^{\circ} \mathrm{C}$. Nach dem Abkühlen goß man auf eine Mischung von $20 \mathrm{ml}$ verd. Salzsäure und $75 \mathrm{ml}$ tiefsiedendem Petrolether, saugte ab und wusch die Titansalze und die Säurephase gut mit Petrolether nach. Die organischen Phasen wurden gewaschen, getrocknet und bei 12 Torr eingedampft. Der feste Rückstand wurde aus Petrolether oder Methanol umkristallisiert: $950 \mathrm{mg}(60 \%)$ farblose Quader, an der Luft allmählich blauviolett, Reinschmp. $155-157^{\circ} \mathrm{C}$. - IR (KBr): $\tilde{v}=3055 \mathrm{~cm}^{-1}, 2960,2920,2858,1689(\mathrm{CN}), 1575,1486,1391,782$, 761. - UV (Cyclohexan): $\lambda_{\max }(\lg \varepsilon)=235 \mathrm{~nm}$ (4.489), 266 (3.519), 273 (3.624), 306 (3.878), 326 (3.784). $-{ }^{1} \mathrm{H}-\mathrm{NMR}\left(\mathrm{CCl}_{4}\right): \delta=1.38$ (sehr br, alle $\mathrm{CH}_{3}$ ), 6.69 (mc, $\left.1 \mathrm{H}\right), 7.03$ (mc, 4H), 7.25 (mc, $\left.4 \mathrm{H}\right), 7.60$ (mc, $2 \mathrm{H}) .-{ }^{1} \mathrm{H}-\mathrm{NMR}\left(\mathrm{CH}_{2} \mathrm{Cl}_{2}\right): \delta=0.78,1.50,1.63,1.70\left(4 \mathrm{CH}_{3}\right)$ bei $-78^{\circ} \mathrm{C}$, koalesziert zu $\delta=1.13\left(1-\mathrm{CH}_{3}\right)$ und $1.67\left(3-\mathrm{CH}_{3}\right), \Delta G^{\mp}$ $=11.3(1) \mathrm{kcal} / \mathrm{mol}$ bei $T_{\mathrm{c}}=-42( \pm 2)^{\circ} \mathrm{C}$; weitere Koaleszenz $\Delta G^{\ddagger}\left(+14^{\circ} \mathrm{C}\right)=14.4 \mathrm{kcal} / \mathrm{mol}$. $-{ }^{1} \mathrm{H}-\mathrm{NMR}$-Linienform (Anisol): 
$\Delta \delta=0.550 \mathrm{ppm}$ temperaturkonstant (Methyl); vgl. Abb. 2. $-{ }^{13} \mathrm{C}-$ NMR-Linienform ( $\mathrm{CDCl}_{3}, 25.15 \mathrm{MHz}$ ): 4 Methylsignale (Tab. 1) koaleszierten bei $-52,-44$ und $-42^{\circ} \mathrm{C}$ zu $\delta=28.8$ und 29.8 (bei $\left.-11^{\circ} \mathrm{C}\right), \Delta G^{*}=10.7(1) \mathrm{kcal} / \mathrm{mol}$; anschließend 4 Koaleszenzen für $1 / 3-\mathrm{CH}_{3}, \mathrm{C}-1 / 3, \mathrm{C}-4 / 7$ und $\mathrm{C}-8 / 9$ zwischen -11 und $+15^{\circ} \mathrm{C}$ (Tab. 2).

$$
\begin{array}{ll}
\mathrm{C}_{23} \mathrm{H}_{23} \mathrm{~N} \text { (313.4) } & \text { Ber. C } 88.13 \text { H } 7.40 \text { N } 4.47 \\
& \text { Gef. C } 88.24 \text { H } 7.20 \text { N } 4.36
\end{array}
$$

2-(Acetylimino)-1,1,3,3-tetramethylindan (3e): Die Lösung von $1.00 \mathrm{~g}(5.34 \mathrm{mmol})$ Imin $3 \mathrm{~d}^{[1]}$ in $10 \mathrm{ml}$ absol. Ether (mit oder ohne $0.68 \mathrm{ml}$ Pyridin) wurde unter Argon mit Eis gekühlt und mit $0.455 \mathrm{ml}(6.4 \mathrm{mmol})$ Acetylchlorid versetzt. Nach $10 \mathrm{~min}$ filtrierte man vom voluminösen Niederschlag (3d-Hydrochlorid ${ }^{\left[{ }^{[1]}\right)}$ ab und goß das Filtrat in $100 \mathrm{ml} 2 \mathrm{~N} \mathrm{NaOH}$, die man dann dreimal ausetherte. Die mit verd. $\mathrm{Na}_{2} \mathrm{CO}_{3}$-Lösung und Wasser gewaschenen Etherextrakte wurden getrocknet und eingedampft. Die Umkristallisation des Rohprodukts $(880 \mathrm{mg})$ aus Hexan bei $-78^{\circ} \mathrm{C}$ lieferte $252 \mathrm{mg} \mathrm{(21 \% )}$ farbloses 3e, Schmp. $88-89^{\circ} \mathrm{C}$. - IR (KBr): $\tilde{v}=$ $2972 \mathrm{~cm}^{-1}, 2929,2866,1712(\mathrm{CO}), 1671(\mathrm{CN}), 1229,762 .-{ }^{1} \mathrm{H}-$ NMR $\left(\mathrm{CCl}_{4}\right): \delta=1.43\left(\mathrm{~s}, 1,3-\mathrm{CH}_{3}\right), 2.16$ (s, Acetyl), $7.10\left(\mathrm{~m}, \mathrm{C}_{6} \mathrm{H}_{4}\right)$. $-{ }^{1} \mathrm{H}-\mathrm{NMR}\left(\mathrm{CDCl}_{3},+9^{\circ} \mathrm{C}\right): \delta=1.45\left(\mathrm{~s}, 1,3-\mathrm{CH}_{3}\right), 2.31$ (s, Acetyl), 7.21 und 7.29 (AA' $\mathrm{BB}^{\prime}$ ), keine Änderung bis $-80^{\circ} \mathrm{C}$ - ${ }^{13} \mathrm{C}-\mathrm{NMR}$ $\left(\mathrm{CDCl}_{3},+9\right.$ oder $\left.+32^{\circ} \mathrm{C}\right): \delta=25.8\left(\mathrm{q},{ }^{1} \mathrm{~J}=128 \mathrm{~Hz}\right.$, Acetyl-CH$)$, 28.7 (qq, ${ }^{1} J=127,{ }^{3} J=4.6 \mathrm{~Hz}, 1,3-\mathrm{CH}_{3}$ ), 48.7 (m, C-1,3), 122.5 $\left(\mathrm{dm},{ }^{1} J=158 \mathrm{~Hz}, \mathrm{C}-4,7\right), 127.8\left(\mathrm{dm},{ }^{1} J=159 \mathrm{~Hz}, \mathrm{C}-5,6\right), 146.2(\mathrm{~m}$, C-8,9), 182.9 (q, ${ }^{2} J=6.1 \mathrm{~Hz}, \mathrm{CO}$, vgl. Lit. ${ }^{[13]}$ ), $185.1\left(\mathrm{~m},{ }^{3} J=4 \mathrm{~Hz}\right.$, $\mathrm{C}-2)$; Koaleszenz bei $-75^{\circ} \mathrm{C}: \Delta \delta=3.28 \mathrm{ppm}$.

$$
\begin{array}{ll}
\mathrm{C}_{15} \mathrm{H}_{19} \mathrm{NO} \text { (229.3) } & \text { Ber. C } 78.56 \text { H } 8.35 \text { N } 6.11 \\
& \text { Gef. C } 78.70 \text { H } 8.24 \text { N } 6.28
\end{array}
$$

1,1,3,3-Tetramethyl-2-/(trimethylsilyl)imino/indan (3g): Aus $406 \mathrm{mg}$ ( $2.17 \mathrm{mmol})$ Imin $3 \mathrm{~d}$ in $4.5 \mathrm{ml}$ wasserfreiem THF wurde die Lithiumverbindung $3 f$ durch Titration mit $1.40 \mathrm{ml}$ Methyllithiumlösung (1.6 $\mathrm{M}$ in Ether, Gasbürette) hergestellt ${ }^{[1]}$. $\mathrm{Zu}$ der gelblich getrübten Lösung gab man $0.330 \mathrm{ml}(2.60 \mathrm{mmol})$ Chlortrimethylsilan und prüfte ${ }^{1} \mathrm{H}-\mathrm{NMR}$-spektroskopisch auf vollständige Umsetzung. Man verdünnte mit ctwas Ether, schüttelte zweimal mit verd. Natronlauge aus und trocknete ohne vorheriges Waschen mit Wasser. Nach Entfernen der Lösungsmittel i.Vak. lagen im Rohprodukt (404 mg) 72\% 3g neben Azin 4 und Imin 3d vor. Es wurde in wasserfreiem Pentan aufgenommen (keinen Ether verwenden) und aus dem Mikrokölbchen destilliert: Mindestens $113 \mathrm{mg}\left(20 \%\right.$ ) farblose Flüssigkeit $3 \mathrm{~g}$, Sdp. $58-60^{\circ} \mathrm{C}$ (Badtemp.)/ 0.005 mbar. - IR (Film): $\tilde{v}=2960 \mathrm{~cm}^{-1}, 1746$ (CN), 1070 (br). ${ }^{1} \mathrm{H}-\mathrm{NMR}\left(\mathrm{CCl}_{4}\right): \delta=0.25\left(\mathrm{~s}, \mathrm{SiMe}_{3}\right), 1.21\left(\mathrm{~s}, 1,3-\mathrm{CH}_{3}\right), 7.08\left(\mathrm{~s}, \mathrm{C}_{6} \mathrm{H}_{4}\right)$. $-{ }^{1} \mathrm{H}$ - und ${ }^{13} \mathrm{C}-\mathrm{NMR}$ (THF/Diethylether $3: 1$ bei $+25^{\circ} \mathrm{C}$ ): Wie Tab. 1, jedoch $\delta=148.2$ für C-8/9 und $\delta$-Werte um $0.3 \mathrm{ppm}$ größer für $1 / 3-\mathrm{CH}_{3}$ und $\mathrm{C}-1 / 3 .-\mathrm{MS}: m / z(\%)=259(37)\left[\mathrm{M}^{+}\right], 244(53)$ $\left[\mathrm{M}-\mathrm{CH}_{3}\right], 160(30), 145(100)$.

$$
\begin{array}{llll}
\mathrm{C}_{16} \mathrm{H}_{25} \mathrm{NSi} \text { (259.5) } & \text { Ber. C } 74.07 \text { H } 9.71 \text { N } 5.40 \\
& \text { Gef. C } 74.01 \text { H 9.56 N } 6.26
\end{array}
$$

2-(Cyanimino)-1,1,3,3-tetramethylindan (3h): Wie oben wurden $900 \mathrm{mg}(4.81 \mathrm{mmol})$ Imin $3 \mathrm{~d}$ in $9 \mathrm{ml}$ wasserfreiem THF mit $3.1 \mathrm{ml}$ Methyllithium $\left(1.6 \mathrm{M}\right.$ in Ether) titriert ${ }^{[1]}$. Dann wurde unter Eiskühlung mit $917 \mathrm{mg}$ (4.81 mmol) 95proz. 4-Toluolsulfonylcyanid versetzt, wobei sofort orange verfärbtes Lithiumtosylat ausfiel. Man goß in $80 \mathrm{ml} 1 \mathrm{~N} \mathrm{NaOH}$, etherte dreimal aus, wusch den Ether neutral und trocknete mit $\mathrm{MgSO}_{4}$. Das feste Rohprodukt $(1.00 \mathrm{~g})$ wurde aus Hexan umkristallisiert: $474 \mathrm{mg}(46 \%)$ schwach gelbe, verwachsene Plättchen, Schmp. $95-97^{\circ} \mathrm{C}$ - IR (KBr): $\tilde{v}=$ $2978 \mathrm{~cm}^{-1}, 2968,2930,2870,2186(\mathrm{CN}), 1639(\mathrm{C}=\mathrm{N}), 1483,768$. $-{ }^{1} \mathrm{H}-\mathrm{NMR}\left(\mathrm{CCl}_{4}\right): \delta=1.45\left(3-\mathrm{CH}_{3}\right), 1.68\left(1-\mathrm{CH}_{3}\right), 7.20$ (verbr. s, $\left.\mathrm{C}_{6} \mathrm{H}_{4}\right)$, temperaturkonstant bis $-49^{\circ} \mathrm{C} ; k=13 \mathrm{~s}^{-1}\left(+23^{\circ} \mathrm{C}\right)$ und
$23 \mathrm{~s}^{-1}\left(+32^{\circ} \mathrm{C}\right)$ durch $\mathrm{CH}_{3}$-Linienformanalyse. - ${ }^{1} \mathrm{H}-\mathrm{NMR}$ $\left(\mathrm{CDCl}_{3},-34^{\circ} \mathrm{C}\right): \delta=1.47\left(\mathrm{~s}, 3-\mathrm{CH}_{3}\right), 1.69\left(\mathrm{~s}, 1-\mathrm{CH}_{3}\right), 7.26,7.29$ und $7.37\left(\mathrm{ABCC}^{\prime}, \mathrm{C}_{6} \mathrm{H}_{4}\right) ; \Delta \delta=0.234 \mathrm{ppm}$ temperaturkonstant bis $-61{ }^{\circ} \mathrm{C}$. Linienformanalyse: $k$ in $\mathrm{s}^{-1} /{ }^{\circ} \mathrm{C}=11(1) /+13,20.5(5) /+19$, $24.5(9) /+21,35(2) /+25,63(3) /+32 .-{ }^{13} \mathrm{C}-\mathrm{NMR}\left(\mathrm{CDCl}_{3}\right): \delta=51.0$ $(\mathrm{C}-1,3), 145.1(\mathrm{C}-8,9), 220.9(\mathrm{C}-2)$ bei $+32^{\circ} \mathrm{C}$, sonst wie bei $-34^{\circ} \mathrm{C}$ (Tab. $1 ; \mathrm{C}-1 / 3, \mathrm{C}-8 / 9$ und $1-/ 3-\mathrm{CH}_{3}$ differenziert durch selektives Entkoppeln ${ }^{[1]}$ ).

$$
\begin{array}{ll}
\mathrm{C}_{14} \mathrm{H}_{16} \mathrm{~N}_{2} \text { (212.3) } & \text { Ber. C } 79.21 \text { H } 7.60 \mathrm{~N} 13.20 \\
& \text { Gef. C } 79.37 \mathrm{H} 7.60 \mathrm{~N} 13.23
\end{array}
$$

1,1,3,3-Tetramethyl-2-/(phenylthio) imino Jindan (3i): Es empfiehlt sich, die Reagenzien in möglichst geringem Überschuß einzusetzen, weil sich sowohl restliches Diphenyldisulfid als auch sein Reaktionsprodukt mit Alkyllithium schlecht von 3i abtrennen lassen. Restliches Imin 3d kann dagegen mit Säure entfernt werden. $\mathrm{Zu}$ $1.280 \mathrm{~g}(6.83 \mathrm{mmol}) 3 \mathrm{~d}$ in $11 \mathrm{ml}$ wasserfreiem THF gab man nach der Titration wie oben mit $4.40 \mathrm{ml}$ Methyllithium ${ }^{[1]}(1.6 \mathrm{M}$ in Ether) $1.565 \mathrm{~g}$ ( $7.17 \mathrm{mmol})$ festes Diphenyldisulfid, das sofort weiterreagierte. Die Aufarbeitung mit Natronlauge und Ether lieferte nach Neutralwaschen und Trocknen $2.090 \mathrm{~g}(103 \%)$ Rohprodukt mit ca. 3\% Diphenyldisulfid. Aus Methanol kristallisierten $1.049 \mathrm{~g} \mathrm{(52 \% )}$ farblose Nadeln, Schmp. $115.5-117^{\circ} \mathrm{C}$.

Mit Benzolsulfenylchlorid ${ }^{[16]}$ erhieit man nur $40 \%$ rohes $3 \mathbf{i}$ aus 3d, weil das dabei verwendete Pyridin weniger basisch als $3 \mathbf{d}$ ist. Aus $\mathrm{CDCl}_{3}$-Lösung wird 3i erst mit konz. Salzsäure extrahiert und dabei teilweise zum Keton hydrolysiert. - IR (KBr): $\tilde{\mathbf{v}}=$ $3060 \mathrm{~cm}^{-1}, 2965,2924,2865,1628(\mathrm{C}=\mathrm{N}$ verbr., w), 1582, 1477 , 757, 735. $-{ }^{1} \mathrm{H}-\mathrm{NMR}\left(\mathrm{CCl}_{4}\right): \delta=1.45\left(3-\mathrm{CH}_{3}\right), 1.63\left(1-\mathrm{CH}_{3}\right), 7.10$ (s, $\left.\mathrm{C}_{6} \mathrm{H}_{5}\right), 7.38\left(\mathrm{mc}, \mathrm{C}_{6} \mathrm{H}_{4}\right) .-{ }^{1} \mathrm{H}$ - und ${ }^{13} \mathrm{C}-\mathrm{NMR}\left(\mathrm{CDCl}_{3}\right)$ : Tab. 1. - Linienformanalyse (Methylprotonen in Diphenylether, $\Delta \delta=$ $0.17 \mathrm{ppm}$ temperaturkonstant): $k$ in s ${ }^{-1} /{ }^{\circ} \mathrm{C}=3.5(5) /+51.2,4.0(5) /$ $+54.5,6.5(5) /+59.2,8.0(5) /+62.5,9.5(5) /+65.8,12.0(5) /+69,21(1) /$ $+72.5,26(1) /+76.3,35(1) /+81,38(3) /+82.5,52(4) /+86.5,80(5) /$ +91; in $\mathrm{CDCl}_{3} 8.0(5) /+62.5$.

$$
\begin{array}{llllll}
\mathrm{C}_{19} \mathrm{H}_{21} \mathrm{NS} \text { (295.4) } & \text { Ber. C } 77.24 \mathrm{H} 7.17 \text { N } 4.74 \text { S } 10.85 \\
& \text { Gef. C } 77.21 \text { H } 7.20 \text { N } 4.44 \text { S } 10.86
\end{array}
$$

1,1,3,3-Tetramethyl-2-[(phenylsulfinyl) imino]indan (3j): Die Lösung von $500 \mathrm{mg}(1.69 \mathrm{mmol}) 3 \mathrm{i}$ und $35 \mathrm{mg}$ Tricaprylammoniumchlorid in $10 \mathrm{ml}$ säurefreiem Chloroform wurde unter Eiskühlung gerührt und mit $985 \mathrm{mg}(1.69 \mathrm{mmol}) 85 \mathrm{proz}$. Magnesiummonoperoxyphthalat ${ }^{[17]}$ (Hexahydrat) in $5 \mathrm{ml}$ dest. Wasser versetzt. Man rührte noch 25 min eisgekühlt und verdünnte dann mit $25 \mathrm{ml}$ $\mathrm{CH}_{2} \mathrm{Cl}_{2}$. Die Phasen wurden getrennt und beide mit $60 \mathrm{ml}$ gesätt. wäßriger $\mathrm{NaHCO}_{3}$-Lösung behandelt, weil der Phasentransferkatalysator sonst die Schichtentrennung beim Ausschütteln erschwert. Der Etherextrakt der wäßrigen Phase wurde mit der $\mathrm{CH}_{2} \mathrm{Cl}_{2}-\mathrm{Lö-}$ sung vereinigt, neutral gewaschen, mit $\mathrm{MgSO}_{4}$ getrocknet und eingedampft. Der Rückstand $(610 \mathrm{mg})$ kristallisierte aus Hexan mit Schmp. $116-117^{\circ} \mathrm{C}$ : $351 \mathrm{mg}(67 \%)$ fast farblose Quader. - IR $(\mathrm{KBr}): \tilde{\mathrm{v}}=2960 \mathrm{~cm}^{-1}, 2935,2860,1637(\mathrm{~s}, \mathrm{C}=\mathrm{N}), 1094(\mathrm{SO}), 1074$, 756. - ${ }^{1} \mathrm{H}-\mathrm{NMR}\left(\mathrm{CDCl}_{3},+9\right.$ oder $\left.+32^{\circ} \mathrm{C}\right): \delta=1.55$ und 1.60 $\left(2 \mathrm{~s}, \mathrm{CH}_{3}\right.$ koalesziert), 7.20 und $7.29\left(\mathrm{AA}^{\prime} \mathrm{BB}^{\prime}, \mathrm{C}_{6} \mathrm{H}_{4}\right), 7.51(\mathrm{~m}, m$ und $p-\mathrm{H}), 7.85(\mathrm{~m}, o-\mathrm{H})$; ähnlich in $\mathrm{CCl}_{4}$ bei $+32^{\circ} \mathrm{C} .-{ }^{13} \mathrm{C}-\mathrm{NMR}$ $\left(\mathrm{CDCl}_{3},+9\right.$ oder $\left.+32^{\circ} \mathrm{C}\right)$ : Tab. 1. Kopplungskonstanten ${ }^{1} J_{\mathrm{CH}} /{ }^{3} J_{\mathrm{CH}}$ (Hz): q $128 / \mathrm{q}$ ca. 5 (1,3-CH $\left.\mathrm{CH}_{3}\right)$ d 159/m (C-4,7), d 164/m (o-C), d 160/ $\mathrm{m}(\mathrm{C}-5,6)$, d $162 / \mathrm{m}(m-\mathrm{C})$, d $162 / \mathrm{t} 7.3(p-\mathrm{C}) ;{ }^{3} J_{\mathrm{CH}}=3.9$ (C-2). ${ }^{1} \mathrm{H}-\mathrm{NMR}\left(\mathrm{CDCl}_{3},-48^{\circ} \mathrm{C}\right): \delta=1.40$ und $1.80\left(2 \mathrm{~s}, 3,1-\mathrm{CH}_{3}\right.$, temperaturkonstant), $1.50\left(\mathrm{~s}, 3-\mathrm{CH}_{3},-0.00107 \mathrm{ppm} / \mathrm{K}\right), 1.62\left(\mathrm{~s}, 1-\mathrm{CH}_{3}\right.$, $+0.000821 \mathrm{ppm} / \mathrm{K})$. Linienformanalyse: $k$ in s ${ }^{-1} /{ }^{\circ} \mathrm{C}=12(2) /-31$, $28(2) /-24,40(3) /-21,600(150) /+9$.

$\mathrm{C}_{19} \mathrm{H}_{21} \mathrm{NOS}$ (311.4) Ber. C 73.27 H 6.80 N $4.50 \mathrm{~S} 10.30$ Gef. C 73.52 H 6.79 N 4.00 S 10.30 
1,1,3,3-Tetramethyl-2-/(phenylsulfonyl) imino /indan $\quad(3 \mathbf{k}): \quad \mathrm{Zu}$ $225 \mathrm{mg}(0.72 \mathrm{mmol}) 3 \mathrm{j}$ in $4 \mathrm{ml}$ ethanol- und säurefreiem Chloroform gab man $147 \mathrm{mg}(0.72 \mathrm{mmol}) 85 \mathrm{proz}, m$-Chlorperbenzoesäure und rührte noch $15 \mathrm{~min}$. Nach Eingießen in $50 \mathrm{ml} 2 \mathrm{~N} \mathrm{Na}_{2} \mathrm{CO}_{3}-$ Lösung wurde mit viermal $15 \mathrm{ml}$ Ether extrahiert und der Etherextrakt neutral gewaschen. Man trocknete mit $\mathrm{MgSO}_{4}$, dampfte ein und isolierte $204 \mathrm{mg}(87 \%)$ Reinprodukt als farblose, feine Spieße mit Schmp. $135-136^{\circ} \mathrm{C}$ (Ethanol). - IR (KBr): $\tilde{v}=$ $2974 \mathrm{~cm}^{-1}, 1633(\mathrm{C}=\mathrm{N}), 1321,1156,745 .-{ }^{1} \mathrm{H}-\mathrm{NMR}\left(\mathrm{CDCl}_{3}\right.$ oder $\left.\mathrm{CCl}_{4}\right): \delta=1.58\left(1,3-\mathrm{CH}_{3}\right), 7.18\left(\mathrm{~m}, \mathrm{C}_{6} \mathrm{H}_{4}\right), 7.48(\mathrm{~m}, m$ - und $p-\mathrm{H})$, $7.95(\mathrm{~m}, o-\mathrm{H}) .-{ }^{13} \mathrm{C}-\mathrm{NMR}\left(\mathrm{CDCl}_{3},+9\right.$ oder $\left.+23^{\circ} \mathrm{C}\right): \delta=28.3$ (qq, $\left.{ }^{1} J=129.5,{ }^{3} J=4.7 \mathrm{~Hz}, 1,3-\mathrm{CH}_{3}\right), 51.1(\mathrm{~m}, \mathrm{C}-1,3), 122.5(\mathrm{dm}$, $\left.{ }^{1} J=159 \mathrm{~Hz}, \mathrm{C}-4,7\right), 126.9\left(\mathrm{dm},{ }^{1} J=168 \mathrm{~Hz}, o-\mathrm{C}\right), 127.9\left(\mathrm{dm},{ }^{1} J=\right.$ $160 \mathrm{~Hz}, \mathrm{C}-5,6), 128.8\left(\mathrm{dm},{ }^{1} J=161 \mathrm{~Hz}, m-\mathrm{C}\right), 132.5\left(\mathrm{dt},{ }^{1} J=162\right.$, ${ }^{3} J=8 \mathrm{~Hz}, p$-C), $141.8\left(\mathrm{t},{ }^{3} J=8 \mathrm{~Hz}\right.$, ipso-C), 145.7 (m, C-8,9), 206.4 (m, C-2); vgl. Tab. 1. - Linienformanalyse (Methylprotonen $\Delta \delta=$ $0.43 \mathrm{ppm}$ temperaturkonstant und ${ }^{13} \mathrm{C}-\mathrm{K}$ oaleszenzen in $\mathrm{CDCl}_{3}$ ): $k$ in $\mathrm{s}^{-1} /{ }^{\circ} \mathrm{C}=22(2) /-48.5,61(2) /-44.5,75(1) /-42,76(4) /-42,90(2) /$ $-39,138(5) /-37,165(5) /-32.5$.

\section{$\mathrm{C}_{19} \mathrm{H}_{21} \mathrm{NO}_{2} \mathrm{~S}(327.4) \quad$ Ber. C $69.69 \mathrm{H} 6.47 \mathrm{~N} 4.28 \mathrm{~S} 9.77$ Gef. C 69.57 H 6.50 N 4.00 S 9.79}

1,1,3,3-Tetramethyl-2-(nitroimino)indan (3I): Nach Literaturangabe ${ }^{[10]}$ wurden $63 \%$ reines 31 in zwei Fraktionen erhalten; Reinschmp. $78-80^{\circ} \mathrm{C}$ (Ethanol, Lit. ${ }^{[10]} 65^{\circ} \mathrm{C},{ }^{1} \mathrm{H}-\mathrm{NMR}$ stark abweichend). $-{ }^{1} \mathrm{H}-\mathrm{NMR}\left(\mathrm{CCl}_{4}\right.$ oder $\left.\mathrm{CDCl}_{3}\right): \delta=1.53$ und $1.55(2 \mathrm{~s}$, 3- und 1- $\left.\mathrm{CH}_{3}\right), 7.16\left(\mathrm{mc}, \mathrm{C}_{6} \mathrm{H}_{4}\right)$. - ${ }^{1} \mathrm{H}-\mathrm{NMR}$ (Diphenylether und Anisol): $\delta=1.35$ und $1.42\left(2 \mathrm{~s}, 3-\right.$ und $\left.1-\mathrm{CH}_{3}\right)$, temperaturkonstant bis $+170^{\circ} \mathrm{C} .-{ }^{13} \mathrm{C}-\mathrm{NMR}\left(\mathrm{CDCl}_{3}\right)$ : Tab. 1 .

\section{$N, N^{\prime}$-Bis (1,1,3,3-tetramethyl-2-indanyliden) hydrazin (4)}

a) Aus $451 \mathrm{mg}$ ( $2.41 \mathrm{mmol}$ ) Imin $3 \mathrm{~d}$ in $4 \mathrm{ml}$ wasserfreiem THF wurde wie oben die Lithiumverbindung $3 \mathrm{f}$ hergestellt und innerhalb von $12 \mathrm{~min} \mathrm{zu}$ einer auf $-78^{\circ} \mathrm{C}$ gekühlten Lösung von $0.464 \mathrm{ml}$ (3.62 mmol) dest. Benzolsulfonylchlorid in $1.5 \mathrm{ml}$ wasserfreiem THF getropft. Die sofort entstandene Suspension wurde aufgewärmt und mit Wasser und Ether aufgearbeitet. Aus Ethanol kristallisierten $119 \mathrm{mg}(27 \%)$ fast farblose Stäbchen mit Schmp. $171-172^{\circ} \mathrm{C}$ (Lit. ${ }^{[14]} 172-173^{\circ} \mathrm{C}$, gelb, 52\%; Lit. ${ }^{[15]} 160-162^{\circ} \mathrm{C}$ ). Beim umgekehrten Zusammengeben der Reaktionspartner erhielt man ebenfalls nur das Azin 4 (8\%), aber nicht $3 \mathbf{k}$.

b) Die Lithiumverbindung $3 \mathrm{f}$ aus $58 \mathrm{mg}(0.31 \mathrm{mmol}) 3 \mathrm{~d}$ in $2 \mathrm{ml}$ wasserfreiem THF reagierte mit $100 \mathrm{mg}(0.31 \mathrm{mmol})$ der $N$-Phenylsulfonyl-Verbindung 3k sofort. Der Rückstand der Aufarbeitung enthielt restliches $3 \mathbf{k}$ und Imin 3d neben 22\% Azin 4, das nach Umkristallisieren aus Cyclohexan und aus Ethanol bei $168-170^{\circ} \mathrm{C}$ schmolz. - IR ( $\mathrm{KBr})$ : Wie Lit. ${ }^{[14,15]}$ - ${ }^{1} \mathrm{H}-\mathrm{NMR}\left(\mathrm{CCl}_{4}\right.$ oder $\left.\mathrm{CDCl}_{3}\right)$ : Tab. 1, wie Lit. ${ }^{[14]}-{ }^{13} \mathrm{C}-\mathrm{NMR}\left(\mathrm{CDCl}_{3}\right)$ : Tab. $1 ;{ }^{1} J_{\mathrm{CH}} /{ }^{3} J_{\mathrm{CH}}(\mathrm{Hz})$ : $\mathrm{q}$ 128/q 4.6 (1,3- $\left.\mathrm{CH}_{3}\right)$, d 156/m (C-4,7), d 160/d 7 (C-5,6). - Mol.Masse (osmometr. in Benzol): Ber. 372.6, gef. 365 .

[1] 14. Mitteilung: R. Knorr, K. Ferchland, J. Mehlstäubl, P. Böhrer, T. P. Hoang, D. S. Stephenson, Chem. Ber. 1992, 125, $2033-2040$, voranstehend.

${ }^{[2]}$ L. M. Jackman, F. A. Cotton, Dynamic NMR Spectroscopy, Academic Press, New York, 1975, S. 244-249.

${ }^{[3]}$ H.-O. Kalinowski, H. Kessler, Top. Stereochem. 1973, 7, 295; besonders S. $332-358$.

${ }^{\left.{ }^{4}\right]}$ C. G. McCarty in The Chemistry of the Carbon-Nitrogen Double Bond (Ed.: S. Patai), Interscience, London, 1970, S. 363-408.

${ }^{[5]}$ R. Knorr, E. Lattke, Tetrahedron Lett. 1977, 3969-3972.

${ }^{[6]}$ E. Lattke, R. Knorr, Chem. Ber. 1981, 114, 1600-1609.

${ }^{[7]}$ R. Knorr, T. von Roman, Angew. Chem. 1984, 96, 349-350; Angew. Chem. Int. Ed. Engl. 1984, 23, 366.

${ }^{[8]}$ D. Y. Curtin, J. W. Hausser, J. Am. Chem. Soc. 1961, 83, $3474-3481$.
${ }^{[9]}$ R. Knorr, K. Polborn, J. Chem. Soc., Perkin Trans. 2, 1991, $1505-1508$.

${ }^{[10]}$ F. S. Guziec, Jr., J. M. Russo, Synthesis 1984, 479-481.

${ }^{[11]}$ S. Jendrzejewski, W. Steglich, Chem. Ber. 1981, 114, 1337-1342, und zitierte Literatur.

${ }^{[12]}$ R. Kupfer, S. Meier, E.-U. Würthwein, Synthesis 1984, 688-690.

${ }^{[13]}$ R. Allmann, R. Kupfer, M. Nagel, E.-U. Würthwein, Chem. Ber. 1984, 117, 1597-1605, und zitierte Literatur.

${ }^{[14]}$ F. S. Guziec, Jr., C. J. Murphy, E. R. Cullen, J. Chem. Soc., Perkin Trans. 1, 1985, 107-113.

${ }^{[15]}$ A. Krebs, W. Rüger, B. Ziegenhagen, M. Hebold, I. Hardtke, R. Müller, M. Schütz, M. Wietzke, M. Wilke, Chem. Ber. 1984, 117, $277-309$.

${ }^{[16]}$ H. Lecher, F. Holschneider, K. Köberle, W. Speer, P. Stöcklin, Chem. Ber. 1925, 58, 409-416.

${ }^{[17]}$ P. Brougham, M. S. Cooper, D. A. Cummerson, H. Heaney, N. Thompson, Synthesis 1987, 1015-1017.

${ }^{[18]}$ G. Binsch, Top. Stereochem. 1968, 3, 97-192.

${ }^{[19]}$ G. Binsch, H. Kessler, Angew. Chem. 1980, 92, 445-463; Angew. Chem. Int. Ed. Engl. 1980, 19, 411.

${ }^{[20]}$ W. B. Jennings, S. Al-Showiman, D. R. Boyd, R. M. Campbell, J. Chem. Soc., Perkin Trans. 2, 1976, $1501-1506$.

${ }^{[21]}$ H. Quast, G. Meichsner, Chem. Ber. 1987, 120, 1049-1058.

${ }^{[22]}$ W. B. Jennings, D. R. Boyd, J. Am. Chem. Soc. 1972, 94, $7187-7188$

${ }^{[23]}$ W. B. Jennings, S. Al-Showiman, M. S. Tolley, D. R. Boyd, J. Chem. Soc., Perkin Trans. 2, 1975, 1535-1539.

${ }^{[24]}$ Eine Unterscheidung zwischen Inversions- und Rotations-Mechanismen wird in dieser Arbeit nicht benötigt.

${ }^{[25]}$ J. P. Idoux, J. A. Sikorski, J. Chem. Soc., Perkin Trans. 2, 1972, $921-923$.

${ }^{[26]}$ H.-O. Kalinowski, H. Kessler, D. Leibfritz, A. Pfeffer, Chem. Ber. 1973, 106, 1023-1032.

${ }^{[27]}$ G. Erker, W. Frömberg, C. Krüger, E. Raabe, J. Am. Chem. Soc. 1988, $110,2400-2405$.

${ }^{[28]}$ J. Bjørgo, D. R. Boyd, W. B. Jennings, P. M. Muckett, L. C. Waring, J. Org. Chem. 1977, 42, 3700-3704.

${ }^{[29]}$ Vgl. Struktur 5 in Lit. ${ }^{[9]}$

${ }^{[30]}$ D. R. Eaton, J. P. K. Tong, Inorg. Chem. 1980, 19, 740-744.

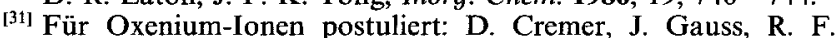
Childs, C. Blackburn, J. Am. Chem. Soc. 1985, 107, 2435-2441.

${ }^{\text {[32] }}$ C.-P. Klages, J. Voß, Chem. Ber. 1980, 113, 2255- 2277.

${ }^{[33]}$ R. van Eldik, T. Asano, W. J. LeNoble, Chem. Rev. 1989, 89, 549-688; T. Asano, W. J. LeNoble, ibid. 1978, 78, 407-489.

${ }^{[34]}$ R. C. Neuman, C. T. Berge, G. A. Binegar, W. Adam, Y. Nishizawa, J. Org. Chem. 1990, 55, 4564-4568.

${ }^{[35]}$ R. van Eldik, H. Kelm, M. Schmittel, C. Rüchardt, J. Org. Chem. 1985, 50, 2998-3000.

${ }^{[36]}$ D. Y. Curtin, E. J. Grubbs, C. G. McCarty, J. Am. Chem. Soc. 1966, $88,2775-2786$.

[37] D. Wurmb-Gerlich, F. Vögtle, A. Mannschreck, H. A. Staab, Liebigs Ann. Chem. 1967, 708, 36-50.

${ }^{[38]}$ Vgl. auch $\mathbf{8 b}$ bei J. J. Worman, E. A. Schmidt, J. Org. Chem. 1970, 35, 2463-2464.

${ }^{[39]}$ R. Knorr, A. Schnegg, Chem. Ber. 1979, 112, 3515-3519.

${ }^{[40]}$ Vgl. jedoch G. W. Buchanan, B. A. Dawson, Org. Magn. Reson. $1980,13,293-298$.

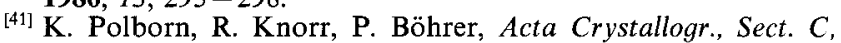
1992, 48, 490-492.

${ }^{[42]}$ W. B. Jennings, S. Al-Showiman, M. S. Tolley, D. R. Boyd, Org. Magn. Reson. 1977, 9, 151-154.

${ }^{\left[{ }^{33}\right]}$ D. R. Boyd, S. Al-Showiman, W. B. Jennings, J. Org. Chem. 1978, 43, 3335-3339.

${ }^{[44]}$ T. A. Hamor, W. B. Jennings, L. D. Proctor, M. S. Tolley, D. R. Boyd, T. Mullan, J. Chem. Soc., Perkin Trans. 2, 1990, 25-30.

${ }^{[45]}$ E.-U. Würthwein, R. Kupfer, S. Meier, M. Krestel, R. Allmann, Chem. Ber. 1988, 121, 591-596.

${ }^{[46]}$ R. J. Cook, K. Mislow, J. Am. Chem. Soc. 1971, 93, 6703-6705.

${ }^{[47]}$ H. Ahlbrecht, E. O. Düber, Synthesis 1982, 273-275.

${ }^{[48]}$ S. Nakahama, A. Hirao, I. Hattori, K. Yamaguchi, Synthesis $1982,461-462$.

[49] J. B. Farmer, R. Snaith, K. Wade, J. Chem. Soc., Dalton Trans. $1972,1501-1502$.

${ }^{[50]}$ R. Knorr, T. von Roman, H. Nöth, S. Böck, J. Chem. Soc., Perkin Trans. 2, 1992, 127-130.

${ }^{[55]}$ W. H. Dawson, D. H. Hunter, C. J. Willis, J. Chem. Soc., Chem. Commun. 1980, 874-875.

[52] P. Luthardt, M. H. Möller, U. Rodewald, E.-U. Würthwein, Chem. Ber. 1989, 122, 1705-1710. 
${ }^{[53]}$ D. J. Hart, K. Kanai, D. G. Thomas, T.-K. Yang, J. Org. Chem. $1983,48,289-294$.

${ }^{[54]}$ C. G. McCarty, D. M. Wieland, Tetrahedron Lett. 1969 , 1787-1790. Vgl. M. Ramezanian, A. B. Padias, F. D. Saeva, H. K. Hall, J. Org. Chem. 1990, 55, $1768-1771$.

${ }^{[55]}$ R. J. Bach, G. J. Wolber, J. Org. Chem. 1982, 47, 239-245.

${ }^{[56]}$ A. Aumüller, S. Hünig, Liebigs Ann. Chem. 1986, 142-164.

${ }^{[57]}$ U. Schubert, S. Hünig, A. Aumüller, Liebigs Ann. Chem. 1985, $1216-1222$.

${ }^{[58]}$ L. Craine, M. Raban, Chem. Rev. 1989, 89, 689-712.

${ }^{[59]}$ F. A. Davis, E. W. Kluger, J. Am. Chem. Soc. 1976, 98, 302- 303.

${ }^{[60]}$ C. Brown, R. F. Hudson, B. T. Grayson, J. Chem. Soc., Chem. Commun. 1978, 156-157.

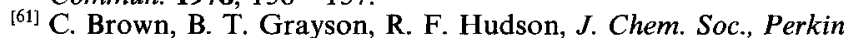
Trans. 2, 1979, 427-434. Vgl. F. A. Davis, W. A. R. Slegeir, J. M. Kaminski, J. Chem. Soc., Chem. Commun. 1972, 634-635.

${ }^{[62]}$ J. M. Klerks, G. van Koten, K. Vrieze, P. Seignette, J. Organomet. Chem. 1981, 219, 9-20.

${ }^{[63]}$ P. D. Robinson, D. H. Hua, J. S. Chen, S. Saha, Acta Crystallogr., Sect. C, 1991, 47, 594-596.

${ }^{[64]}$ F. A. Davis, A. J. Friedman, E. W. Kluger, J. Am. Chem. Soc. 1974, 96, 5000-5001.

${ }^{[65]}$ M. Raban, E. Carlson, J. Am. Chem. Soc. 1971, 93, 685-691.

${ }^{\left[{ }^{66]}\right.}$ F. Vögtle, A. Mannschreck, H. A. Staab, Liebigs Ann. Chem. 1967, $708,51-56$

\section{CAS-Registry-Nummern}

3a: 142294-67-5 / 3b: 89929-48-6 / 3c: 142396-60-9 / 3d: $92807-$ 11-9 / 3e: 142396-61-0 / 3g: 142396-66-5 / 3h: 142396-62-1 / 3i: 142396-63-2 / 3j: 142396-64-3 / 3k: 142396-65-4 / 31: 92807-07-3 /
${ }^{[67]}$ S. Adamopoulos, A. J. Boulton, R. Tadayoni, G. A. Webb, $J$. Chem. Soc., Perkin Trans. 1, 1987, 2073-2077.

${ }^{[68]}$ G. Büchi, H. Wüest, J. Org. Chem. 1979, 44, 4116-4120.

${ }^{[69]}$ F. Bondavalli, P. Schenone, Synthesis 1979, 830-832.

${ }^{[70]}$ F. C. Brown, D. G. Morris, J. Chem. Soc., Perkin Trans. 2, 1977, $125-127$.

[71] J. Burdon, A. Ramirez, Tetrahedron 1973, 29, 4195-4198.

${ }^{[72]}$ T. S. Cameron, R. E. Cordes, D. G. Morris, A. M. Murray, $J$. Chem. Soc., Perkin Trans. 2, 1979, 300-303.

${ }^{[73]}$ J. P. Ritchie, J. Am. Chem. Soc. 1989, 111, 2517-2520.

${ }^{[74]}$ Neue Zusammenfassung: C. Hansch, A. Leo, R. W. Taft, Chem. Rev. 1991, 91, 165-195.

${ }^{[75]}$ R. Knorr, T.-P. Hoang, J. Mehlstäubl, M. Hintermeyer-Hilpert, H.-D. Lüdemann, E. Lang, G. Sextl, W. Rattay, P. Böhrer, in Vorbereitung.

${ }^{[76]}$ M. Kontoyianni, A. J. Hoffman, J. P. Bowen, J. Computat. Chem. 1992, 13, 57-65.

${ }^{[77]}$ K. Takeuchi, Y. Ohga, T. Kitagawa, J. Org. Chem. 1991, 56, $5007-5008$.

${ }^{[78]}$ G. Völkel, E. Lang, H.-D. Lüdemann, Ber. Bunsenges. Phys. Chem. 1979, $83,722-729$.

4: 89050-92-0 / $\alpha$-Naphthylamin: 134-32-7 / 1,1,3,3-Tetramethyl2-indanon: 5689-12-3 / 4-Toluolsulfonylcyanid: 19158-51-1 / Diphenyldisulfid: $882-33-7$ 